\title{
Energies and structure of additive sets
}

\author{
Shkredov Ilya * \\ Steklov Mathematical Institute \\ ul. Gubkina, 8 \\ 119991, Moscow, Russia \\ IITP RAS \\ Bolshoy Karetny per., 19 \\ 127994, Moscow, Russia \\ ilya.shkredov@gmail.com
}

Submitted: May 14, 2014; Accepted: Sep 6, 2014; Published: Sep 18, 2014

Mathematics Subject Classifications: 11B13, 11B30

\begin{abstract}
In this paper we prove that any sumset or difference set has large $E_{3}$ energy. Also, we give a full description of families of sets having critical relations between some kind of energies such as $\mathrm{E}_{k}, \mathrm{~T}_{k}$ and Gowers norms. In particular, we give criteria for a set to be a

- set of the form $H \dot{+} \Lambda$, where $H+H$ is small and $\Lambda$ has "random structure",

- set equal to a disjoint union of sets $H_{j}$ each with small doubling,

- set having a large subset $A^{\prime}$ with $2 A^{\prime}$ equal to a set with small doubling and $\left|A^{\prime}+A^{\prime}\right| \approx|A|^{4} / \mathrm{E}(A)$.
\end{abstract}

Keywords: additive combinatorics; additive energy; sumsets; Gowers norms

\section{Introduction}

Let $\mathbf{G}=(\mathbf{G},+)$ be an abelian group. For two sets $A, B \subseteq \mathbf{G}$ define the sumset as

$$
A+B:=\{x \in \mathbf{G}: x=a+b, a \in A, b \in B\}
$$

and, similarly, the difference set

$$
A-B:=\{x \in \mathbf{G}: x=a-b, a \in A, b \in B\} .
$$

*Supported by Russian Scientific Foundation grant RSF 14-11-00433. 
Also denote the additive energy of a set $A$ by

$$
\mathrm{E}(A)=\mathrm{E}_{2}(A)=\left|\left\{a_{1}-a_{1}^{\prime}=a_{2}-a_{2}^{\prime}: a_{1}, a_{1}^{\prime}, a_{2}, a_{2}^{\prime} \in A\right\}\right|,
$$

and $\mathrm{E}_{k}(A)$ energy as

$$
\mathrm{E}_{k}(A)=\left|\left\{a_{1}-a_{1}^{\prime}=a_{2}-a_{2}^{\prime}=\cdots=a_{k}-a_{k}^{\prime}: a_{1}, a_{1}^{\prime}, \ldots, a_{k}, a_{k}^{\prime} \in A\right\}\right| .
$$

The special case $k=1$ gives us $\mathrm{E}_{1}(A)=|A|^{2}$ because there is no any restriction in the set from (1). So, the cardinality of a set can be considered as a degenerate sort of energy. Note that a trivial upper bound for $\mathrm{E}_{k}(A)$ is $|A|^{k+1}$. Now recall a well-known Balog-Szemerédi-Gowers Theorem [37].

Theorem 1. Let $A \subseteq \mathbf{G}$ be a set, and $K \geqslant 1$ be a real number. Suppose that $\mathrm{E}(A) \geqslant$ $|A|^{3} / K$. Then there is $A^{\prime} \subseteq A$ such that $\left|A^{\prime}\right| \gg|A| / K^{C}$ and

$$
\left|A^{\prime}-A^{\prime}\right| \ll K^{C}\left|A^{\prime}\right|,
$$

where $C>0$ is an absolute constant.

So, Balog-Szemerédi-Gowers Theorem can be considered as a result about the structure of sets $A$ having the extremal (in terms of its cardinality or $\mathrm{E}_{1}(A)$ in other words) value of $\mathrm{E}(A)$. Namely, any of such a set has a subset $A^{\prime}$ with the extremal value of cardinality of its difference set. These sets $A^{\prime}$ are called sets with small doubling. On the other hand, it is easy to obtain, using the Cauchy-Schwarz inequality, that any $A$ having subset $A^{\prime}$ such that (2) holds, automatically has polynomially large energy $\mathrm{E}(A) \gg_{K}|A|^{3}$ (see e.g. [37]). Moreover, the structure of sets with small doubling is known more or less thanks to a well-known Freiman's theorem (see [37] or [25]). Thus, Theorem 1 finds subsets of $A$ with rather rigid structure and, actually, it is a criterium for a set $A$ to be a set with large (in terms of $\mathrm{E}_{1}(A)$ ) additive energy: $\mathrm{E}(A) \sim_{K}|A|^{3} \sim_{K}\left(\mathrm{E}_{1}(A)\right)^{3 / 2}$.

In the paper we consider another extremal relations between different energies and describe the structure of sets having these critical relations. Such kind of theorems have plenty of applications. It is obvious for Balog-Szemerédi-Gowers theorem, see e.g. [37], [3], [14], [15], [19], [21] and so on; for recent applications using critical relations between energies $\mathrm{E}_{2}(A)$ and $\mathrm{E}_{3}(A)$, see e.g. [32], [33] and others. Before formulate our main results let us recall a beautiful theorem of Bateman-Katz [3], [4] which is another example of theorems are called "structural" by us.

Theorem 2. Let $A \subseteq \mathbf{G}$ be a symmetric set, $\tau_{0}, \sigma_{0}$ be nonnegative real numbers and $A$ has the property that for any $A_{*} \subseteq A,\left|A_{*}\right| \gg|A|$ the following holds $\mathrm{E}\left(A_{*}\right) \gg \mathrm{E}(A)=|A|^{2+\tau_{0}}$. Suppose that $\mathrm{T}_{4}(A) \ll|A|^{4+3 \tau_{0}+\sigma_{0}}$. Then there exists a function $f_{\tau_{0}}:(0,1) \rightarrow(0, \infty)$ with $f_{\tau_{0}}(\eta) \rightarrow 0$ as $\eta \rightarrow 0$ and a number $0 \leqslant \alpha \leqslant \frac{1-\tau_{0}}{2}$ such that there are sets $X_{j}, H_{j} \subseteq \mathbf{G}$, $B_{j} \subseteq A, j \in\left[|A|^{\alpha-f_{\tau_{0}}\left(\sigma_{0}\right)}\right]$ with

$$
\begin{gathered}
\left|H_{j}\right| \ll|A|^{\tau_{0}+\alpha+f_{\tau_{0}}\left(\sigma_{0}\right)}, \quad\left|X_{j}\right| \ll|A|^{1-\tau_{0}-2 \alpha+f_{\tau_{0}}\left(\sigma_{0}\right)}, \\
\left|H_{j}-H_{j}\right| \ll\left|H_{j}\right|^{1+f_{\tau_{0}}\left(\sigma_{0}\right)}, \\
\left|\left(X_{j}+H_{j}\right) \cap B_{j}\right| \gg|A|^{1-\alpha-f_{\tau_{0}}\left(\sigma_{0}\right)},
\end{gathered}
$$

and $B_{i} \cap B_{j}=\emptyset$ for all $i \neq j$. 
Here $\mathrm{T}_{4}(A)$ is the number of solutions of the equation $a_{1}+a_{2}+a_{3}+a_{4}=a_{1}^{\prime}+a_{2}^{\prime}+a_{3}^{\prime}+a_{4}^{\prime}$, $a_{1}, a_{2}, a_{3}, a_{4}, a_{1}^{\prime}, a_{2}^{\prime}, a_{3}^{\prime}, a_{4}^{\prime} \in A$ and this characteristic is another sort of energy. One can check that any set satisfying (3)-(5), $\mathrm{E}(A)=|A|^{2+\tau_{0}}$ and all another conditions of the theorem is an example of a set having $\mathrm{T}_{4}(A) \ll|A|^{4+3 \tau_{0}+\sigma_{0}}$. Note that if $\mathrm{E}(A)=|A|^{2+\tau_{0}}$ then by the Hölder inequality one has $\mathrm{T}_{4}(A) \geqslant|A|^{4+3 \tau_{0}}$. Thus, Theorem 2 gives us a full description of sets having critical relations between a pair of two energies: $\mathrm{E}(A)$ and $\mathrm{T}_{4}(A)$.

There are two opposite extremal cases in Theorem $2: \alpha=0$ and $\alpha=\frac{1-\tau_{0}}{2}$. For simplicity consider the situation when $\mathbf{G}=\mathbb{F}_{2}^{n}$. In the case $\alpha=0$ by Bateman-Katz result our set $A$, roughly speaking, is close to a set of the form $H \dot{+} \Lambda$, where $\dot{+}$ means the direct sum, $H \subseteq \mathbf{F}_{2}^{n}$ is a subspace, and $\Lambda \subseteq \mathbf{F}_{2}^{n}$ is a dissociated set (basis), $|\Lambda| \sim$ $|A| /|H| \sim|A|^{1-\tau_{0}}$. These sets are interesting in its own right being counterexamples in many problems of additive combinatorics. The reason for this is trivial. Indeed, the sets have rather mixed properties: on the one hand they contain translations $H+\lambda, \lambda \in \Lambda$ of really structured set $H$ but on the other hand they have also some random properties, for example, its Fourier coefficients (see the definition in section 2) are small. Our first result says that a set $A$ is close to a set of the form $H \dot{+} \Lambda$ iff there is the critical relation between $\mathrm{E}_{3}(A)$ and $\mathrm{E}(A)$, that is $\mathrm{E}_{3}(A) \gg|A| \mathrm{E}(A)$. For more rigorous formulation see Theorem 22.

In the situation $\alpha=\frac{1-\tau_{0}}{2}, \mathbf{G}=\mathbb{F}_{2}^{n}$ our set $A$ looks like a union of (additively) disjoint subspaces $H_{1}, \ldots, H_{k}$ (see example (iii) from [26]) with $k=|A|^{\frac{1+\tau_{0}}{2}}$. Such sets can be called self-dual sets, see [33]. In our second result we show that, roughly speaking, any such a set has critical relation between $\mathrm{E}_{3}(A)$ (more precisely, the product $\mathrm{E}(A) \cdot \mathrm{E}_{4}(A)$ ) and so-called Gowers $U^{3}$-norm of the set $A$ (see the definition in section 7 ) and vice versa. Theorem 46 contains the exact formulation.

These two structural results on sets having critical relations between a pair of its energies are the hearth of our paper. In the opposite of Theorem 2 almost all bounds of the paper are polynomial, excluding, of course, the dependence on the number $k$ of the considered energies $\mathrm{E}_{k}, \mathrm{~T}_{k}$ or $U^{k}$ if its appear. Moreover the first structural theorem hints us a partial answer to the following important question. Consider the difference set $D=A-A$ or the sumset $S=A+A$ of an arbitrary set $A$. What can we say nontrivial about the energies of $D, S$ in terms of the energies of $A$ ? In view of the first example above, that is $\mathbf{G}=\mathbb{F}_{2}^{n}, A=H \dot{+} \Lambda,|\Lambda|=K, \mathrm{E}(A) \sim|A|^{3} / K$ we cannot hope to obtain a nontrivial bound for additive energy of $D$ or $S$ because in the case $D=S=H \dot{+}(\Lambda+\Lambda)$, and hence it has a similar structure to $A$ with $\Lambda$ replacing by $\Lambda+\Lambda$. On the other hand, we know that the sets of the form $H \dot{+} \Lambda$ have large $\mathrm{E}_{3}$ energy. Thus, one can hope to obtain a good lower bound for $\mathrm{E}_{3}(D)$ and $\mathrm{E}_{3}(S)$. It turns out to be the case and we prove it in section 6. Roughly speaking, our result asserts that if $|D|=K|A|, \mathrm{E}(A) \ll|A|^{3} / K$ then

$$
\mathrm{E}_{3}(D) \gg K^{7 / 4}|A|^{4},
$$

and a similar inequality for $A+A$.

The paper is organized as follows. We start with definitions and notations used in the paper. In the next section we give several characterisations of sets of the form $A=H \dot{+} \Lambda$, 
where $H$ is a set with small doubling, $\Lambda$ is a "dissociated" set. Also we consider a "dual" question on sets having critical relations between $\mathrm{T}_{4}$ and $\mathrm{E}$ energies, that is the situation when $\mathrm{T}_{4}(A)$ is large in terms on $\mathrm{E}(A)$. It was proved that, roughly speaking, $A$ contains a large subset $A^{\prime}$ such that the sequence $A^{\prime}, 2 A^{\prime}, 3 A^{\prime}, \ldots$ is stabilized at the second step, namely, $A^{\prime}+A^{\prime}$ is a set with small doubling and, besides, $\left|A^{\prime}+A^{\prime}\right| \approx|A|^{4} / \mathrm{E}(A)$ in the only case when $\mathrm{T}_{4}(A) \gg|A|^{2} \mathrm{E}(A)$, see Theorem 23 .

Section 6 contains the proof of inequality (6) and we make some preliminaries to this in section 5. For example, we obtain in the section an interesting characterisation of sumsets $S=A+A$ or difference sets $D=A-A$ with extremal cardinalities of intersections

$$
|A| \leqslant\left|D \cap\left(D+x_{1}\right) \cap \cdots \cap\left(D+x_{s}\right)\right| \leqslant|A|^{1+o(1)},
$$

and, similarly, for $S$, see Theorem 28. It turns out that for such sets $D, S$ the set $A$ should have either very small $O\left(|A|^{k+o(1)}\right)$ energy $\mathrm{E}_{k}(A)$ or very large $\gg|A|^{3-o(1)}$ additive energy. In other words either $A$ has "random behaviour" or, in contrary, is very structured. Clearly, both situations are realized: the first one in the situation when $A$ is a fair random set (and hence $A \pm A$ has almost no structure) and the second one if $A$ is a set with small doubling, say.

In section 7 we consider some simple properties of Gowers norms of the characteristic function of a set $A$ and prove a preliminary result on the connection of $\mathrm{E}(A)$ with $\mathrm{E}(A \cap$ $(A+s)), s \in A-A$, see Theorem 39. It gives a partial counterexample to a famous construction of Gowers [14], [15] of uniform sets with non-uniform intersections $\mathrm{E}(A \cap(A+$ s)) (see the definitions in [14], [15] or [37]). We show that although all sets $A \cap(A+s)$ can be non-uniform but there is always $s \neq 0$ such that $\mathrm{E}(A \cap(A+s)) \ll|A \cap(A+s)|^{3-c}$, $c>0$, provided by some weak conditions take place. This question was asked to the author by T. Schoen.

In the next section we develop the investigation from the previous one and characterize all sets with critical relation between Gowers $U^{3}$-norm and the energies $\mathrm{E}, \mathrm{E}_{4}$ or $\mathrm{E}_{3}$. Also we consider some questions on finding in $A$ a family of disjoint sets $A \cap(A+s)$ or its large disjoint subsets.

A lot of results of the paper such as Bateman-Katz theorem are proved under some regular conditions on $A$. For example, the assumption from Theorem 2 require that for all $A_{*} \subseteq A,\left|A_{*}\right| \gg|A|$ the following holds $\mathrm{E}\left(A_{*}\right) \gg \mathrm{E}(A)$. We call the conditions as connectedness of our set $A$ (see the definitions from sections 3,7 ) and prove in the appendix that any set contains some large connected subset. Basically, we generalize the method from [31].

Thus, we have characterized two extremal situations of Theorem 2 in terms of energies. Is there some similar characterisation for other cases of the result? Do exist criteria in terms of energies for another families of sets? Finally, are there further characteristics of sumsets/difference sets which separate it from arbitrary sets? 


\section{Definitions}

Let $\mathbf{G}$ be an abelian group. If $\mathbf{G}$ is finite then denote by $N$ the cardinality of $\mathbf{G}$. It is well-known [23] that the dual group $\widehat{\mathbf{G}}$ is isomorphic to $\mathbf{G}$ in the case. Let $f$ be a function from $\mathbf{G}$ to $\mathbb{C}$. We denote the Fourier transform of $f$ by $\widehat{f}$,

$$
\widehat{f}(\xi)=\sum_{x \in \mathbf{G}} f(x) e(-\xi \cdot x)
$$

where $e(x)=e^{2 \pi i x}$ and $\xi$ is a homomorphism from $\widehat{\mathbf{G}}$ to $\mathbb{R} / \mathbb{Z}$ acting as $\xi: x \rightarrow \xi \cdot x$. We rely on the following basic identities

$$
\begin{gathered}
\sum_{x \in \mathbf{G}}|f(x)|^{2}=\frac{1}{N} \sum_{\xi \in \widehat{\mathbf{G}}}|\widehat{f}(\xi)|^{2}, \\
\sum_{y \in \mathbf{G}}\left|\sum_{x \in \mathbf{G}} f(x) g(y-x)\right|^{2}=\frac{1}{N} \sum_{\xi \in \widehat{\mathbf{G}}}|\widehat{f}(\xi)|^{2}|\widehat{g}(\xi)|^{2},
\end{gathered}
$$

and

$$
f(x)=\frac{1}{N} \sum_{\xi \in \widehat{\mathbf{G}}} \widehat{f}(\xi) e(\xi \cdot x) .
$$

If

$$
(f * g)(x):=\sum_{y \in \mathbf{G}} f(y) g(x-y) \quad \text { and } \quad(f \circ g)(x):=\sum_{y \in \mathbf{G}} f(y) g(y+x)
$$

then

$$
\widehat{f * g}=\widehat{f} \widehat{g} \quad \text { and } \quad \widehat{f \circ g}=\widehat{f} c \widehat{g}=\overline{\bar{f}} \widehat{g},
$$

where for a function $f: \mathbf{G} \rightarrow \mathbb{C}$ we put $f^{c}(x):=f(-x)$. Clearly, $(f * g)(x)=(g * f)(x)$ and $(f \circ g)(x)=(g \circ f)(-x), x \in \mathbf{G}$. The $k$-fold convolution, $k \in \mathbb{N}$ we denote by $*_{k}$, so $*_{k}:=*\left(*_{k-1}\right)$.

We use in the paper the same letter to denote a set $S \subseteq \mathbf{G}$ and its characteristic function $S: \mathbf{G} \rightarrow\{0,1\}$. Clearly, $S$ is the characteristic function of a set iff

$$
\widehat{S}(x)=N^{-1}(\widehat{\widehat{S}} \circ \widehat{S})(x) .
$$

Write $\mathrm{E}(A, B)$ for the additive energy of two sets $A, B \subseteq \mathbf{G}$ (see e.g. [37]), that is

$$
\mathrm{E}(A, B)=\left|\left\{a_{1}+b_{1}=a_{2}+b_{2}: a_{1}, a_{2} \in A, b_{1}, b_{2} \in B\right\}\right| .
$$

If $A=B$ we simply write $\mathrm{E}(A)$ instead of $\mathrm{E}(A, A)$. Clearly,

$$
\mathrm{E}(A, B)=\sum_{x}(A * B)(x)^{2}=\sum_{x}(A \circ B)(x)^{2}=\sum_{x}(A \circ A)(x)(B \circ B)(x) .
$$


and by (9),

$$
\mathrm{E}(A, B)=\frac{1}{N} \sum_{\xi}|\widehat{A}(\xi)|^{2}|\widehat{B}(\xi)|^{2}
$$

Let

$$
\mathrm{T}_{k}(A):=\left|\left\{a_{1}+\cdots+a_{k}=a_{1}^{\prime}+\cdots+a_{k}^{\prime}: a_{1}, \ldots, a_{k}, a_{1}^{\prime}, \ldots, a_{k}^{\prime} \in A\right\}\right|=\frac{1}{N} \sum_{\xi}|\widehat{A}(\xi)|^{2 k}
$$

and more generally

$$
\mathrm{T}_{k}\left(A_{1}, \ldots, A_{k}\right):=\left|\left\{a_{1}+\cdots+a_{k}=a_{1}^{\prime}+\cdots+a_{k}^{\prime}: a_{1}, a_{1}^{\prime} \in A_{1}, \ldots, a_{k}, a_{k}^{\prime} \in A_{k}\right\}\right| .
$$

Let also

$$
\sigma_{k}(A):=\left(A *_{k} A\right)(0)=\left|\left\{a_{1}+\cdots+a_{k}=0: a_{1}, \ldots, a_{k} \in A\right\}\right| .
$$

Notice that for a symmetric set $A$ that is $A=-A$ one has $\sigma_{2}(A)=|A|$ and $\sigma_{2 k}(A)=$ $\mathrm{T}_{k}(A)$. Having a set $P \subseteq A-A$ we write $\sigma_{P}(A):=\sum_{x \in P}(A \circ A)(x)$.

For a sequence $s=\left(s_{1}, \ldots, s_{k-1}\right)$ put $A_{s}^{B}=B \cap\left(A-s_{1}\right) \cdots \cap\left(A-s_{k-1}\right)$. If $B=A$ then write $A_{s}$ for $A_{s}^{A}$. Let

$$
\mathrm{E}_{k}(A)=\sum_{x \in \mathbf{G}}(A \circ A)(x)^{k}=\sum_{s_{1}, \ldots, s_{k-1} \in \mathbf{G}}\left|A_{s}\right|^{2}
$$

and

$$
\mathrm{E}_{k}(A, B)=\sum_{x \in \mathbf{G}}(A \circ A)(x)(B \circ B)(x)^{k-1}=\sum_{s_{1}, \ldots, s_{k-1} \in \mathbf{G}}\left|B_{s}^{A}\right|^{2}
$$

be the higher energies of $A$ and $B$. The second formulas in (15), (16) can be considered as the definitions of $\mathrm{E}_{k}(A), \mathrm{E}_{k}(A, B)$ for non integer $k, k \geqslant 1$. Similarly, we write $\mathrm{E}_{k}(f, g)$ for any complex functions $f, g$ and more generally

$$
\mathrm{E}_{k}\left(f_{1}, \ldots, f_{k}\right)=\sum_{x}\left(f_{1} \circ f_{1}\right)(x) \ldots\left(f_{k} \circ f_{k}\right)(x) .
$$

Putting $\mathrm{E}_{1}(A)=|A|^{2}$. For a set $P \subseteq \mathbf{G}$ write $\mathrm{E}_{k}^{P}(A):=\sum_{s \in P}\left|A_{s}\right|^{k}, \mathrm{E}^{P}(A):=\mathrm{E}_{2}^{P}(A)$. We put $\mathrm{E}_{k}^{*}(A)$ for $\mathrm{E}_{k}^{*}(A)=\sum_{s \neq 0}\left|A_{s}\right|^{k}$.

Clearly,

$$
\begin{aligned}
\mathrm{E}_{k+1}(A, B) & =\sum_{x}(A \circ A)(x)(B \circ B)(x)^{k} \\
& =\sum_{x_{1}, \ldots, x_{k-1}}\left(\sum_{y} A(y) B\left(y+x_{1}\right) \ldots B\left(y+x_{k}\right)\right)^{2}=\mathrm{E}\left(\Delta_{k}(A), B^{k}\right),
\end{aligned}
$$

where

$$
\Delta(A)=\Delta_{k}(A):=\left\{(a, a, \ldots, a) \in A^{k}\right\} .
$$

We also put $\Delta(x)=\Delta(\{x\}), x \in \mathbf{G}$.

Quantities $\mathrm{E}_{k}(A, B)$ can be written in terms of generalized convolutions. 
Definition 3. Let $k \geqslant 2$ be a positive number, and $f_{0}, \ldots, f_{k-1}: \mathbf{G} \rightarrow \mathbb{C}$ be functions. Denote by

$$
\mathcal{C}_{k}\left(f_{0}, \ldots, f_{k-1}\right)\left(x_{1}, \ldots, x_{k-1}\right)
$$

the function

$$
\mathcal{C}_{k}\left(f_{0}, \ldots, f_{k-1}\right)\left(x_{1}, \ldots, x_{k-1}\right)=\sum_{z} f_{0}(z) f_{1}\left(z+x_{1}\right) \ldots f_{k-1}\left(z+x_{k-1}\right) .
$$

Thus, $\mathcal{C}_{2}\left(f_{1}, f_{2}\right)(x)=\left(f_{1} \circ f_{2}\right)(x)$. If $f_{1}=\cdots=f_{k}=f$ then write $\mathcal{C}_{k}(f)\left(x_{1}, \ldots, x_{k-1}\right)$ for $\mathcal{C}_{k}\left(f_{1}, \ldots, f_{k}\right)\left(x_{1}, \ldots, x_{k-1}\right)$.

In particular, $\left(\Delta_{k}(B) \circ A^{k}\right)\left(x_{1}, \ldots, x_{k}\right)=\mathcal{C}_{k+1}(B, A, \ldots, A)\left(x_{1}, \ldots, x_{k}\right), k \geqslant 1$.

Quantities $\mathrm{E}_{k}(A)$ and $\mathrm{T}_{k}(A)$ are "dual" in some sense. For example in [33], Note 6.6 (see also [28]) it was proved that

$$
\left(\frac{\mathrm{E}_{3 / 2}(A)}{|A|}\right)^{2 k} \leqslant \mathrm{E}_{k}(A) \mathrm{T}_{k}(A),
$$

provided by $k$ is even. Moreover, from $(7)-(10),(12)$ it follows that $\tilde{\mathrm{E}}_{2 k}(\widehat{A}):=\sum_{x}(\overline{\widehat{A}} \circ$ $\widehat{A})^{k}(x)(\widehat{A} \circ \widehat{\widehat{A}})^{k}(x)=N^{2 k+1} \mathrm{~T}_{k}(A)$ and $\mathrm{T}_{k}\left(|\widehat{A}|^{2}\right)=N^{2 k-1} \mathrm{E}_{2 k}(A)$.

For a positive integer $n$, we set $[n]=\{1, \ldots, n\}$. Let $x$ be a vector. By $\|x\|$ denote the number of components of $x$. All logarithms are to base 2 . Signs $\ll$ and $\gg$ are the usual Vinogradov's symbols and if the bounds depend on some parameter $M$ polynomially then we write $\ll_{M}, \gg_{M}$. If for two numbers $a, b$ the following holds $a \ll_{M} b, b \ll_{M} a$ then we write $a \sim_{M} b$. In particular, $a \sim b$ means $a \ll b$ and $b \ll a$.

All polynomial bounds in the paper can be obtained in explicit way.

\section{Preliminaries}

Let us begin with the famous Plünnecke-Ruzsa inequality (see [22] or [37], e.g.).

Lemma 4. Let $A \subseteq \mathbf{G}$ be a set. Then for all positive integers $n, m$ the following holds

$$
|n A-m A| \leqslant K^{n+m}|A| \text {. }
$$

We need in several quantitative versions of the Balog-Szemerédi-Gowers Theorem. The first symmetric variant is due to T. Schoen [27].

Theorem 5. Let $A \subseteq \mathbf{G}$ be a set, $K \geqslant 1$ and $\mathrm{E}(A) \geqslant \frac{|A|^{3}}{K}$. Then there is $A^{\prime} \subseteq A$ such that

$$
\left|A^{\prime}\right| \gg \frac{|A|}{K}
$$

and

$$
\left|A^{\prime}-A^{\prime}\right| \ll K^{4}\left|A^{\prime}\right|
$$


Also we need in a version of Balog-Szemerédi-Gowers theorem in the asymmetric form, see [37], Theorem 2.35 .

Theorem 6. Let $A, B \subseteq \mathbf{G}$ be two sets, $|B| \leqslant|A|$, and $M \geqslant 1$ be a real number. Let also $L=|A| /|B|$ and $\varepsilon \in(0,1]$ be a real parameter. Suppose that

$$
\mathrm{E}(A, B) \geqslant \frac{|A||B|^{2}}{M} .
$$

Then there are two sets $H \subseteq \mathbf{G}, \Lambda \subseteq \mathbf{G}$ and $z \in \mathbf{G}$ such that

$$
\begin{gathered}
|(H+z) \cap B| \gg_{\varepsilon} M^{-O_{\varepsilon}(1)} L^{-\varepsilon}|B|, \quad|\Lambda| \ll_{\varepsilon} M^{O_{\varepsilon}(1)} L^{\varepsilon} \frac{|A|}{|H|}, \\
|H-H| \ll_{\varepsilon} M^{O_{\varepsilon}(1)} L^{\varepsilon} \cdot|H|,
\end{gathered}
$$

and

$$
|A \cap(H+\Lambda)| \gg_{\varepsilon} M^{-O_{\varepsilon}(1)} L^{-\varepsilon}|A|
$$

The next lemma is a special case of Lemma 2.8 from [34]. In particular, it gives us a connection between $\mathrm{E}_{3}(A)$ and $\mathrm{E}\left(A, A_{s}\right)$, see e.g [28].

Lemma 7. Let $A \subseteq \mathbf{G}$ be a set. Then for every $k, l \in \mathbb{N}$

$$
\sum_{\substack{s, t: \\\|s\|=k-1,\|t\|=l-1}} \mathrm{E}\left(A_{s}, A_{t}\right)=\mathrm{E}_{k+l}(A) .
$$

In particular,

$$
\mathrm{E}_{3}(A)=\sum_{s} \mathrm{E}\left(A, A_{s}\right)
$$

Now recall a lemma from [30], [33].

Lemma 8. Let $A$ be a subset of an abelian group, $Q \subseteq A-A$. Then

$$
\sum_{s \in Q}\left|A \pm A_{s}\right| \geqslant \frac{\sigma_{Q}^{2}(A)|A|^{2}}{\mathrm{E}_{3}(A)}
$$

and

$$
\mathrm{E}_{3}(Q, A, A) \cdot \mathrm{E}_{3}(A) \geqslant \frac{\mathrm{E}^{2}(A) \sigma_{Q}^{4}(A)}{|A|^{6}}
$$

Let also give a simple Corollary 18 from [32]. 
Lemma 9. Let $A \subseteq \mathbf{G}$ be a set. Then

$$
\sum_{s} \frac{\left|A_{s}\right|^{2}}{\left|A \pm A_{s}\right|} \leqslant \frac{\mathrm{E}_{3}(A)}{|A|^{2}}
$$

We give a small generalization of Proposition 11 from [28], see also [21].

Lemma 10. Let $A \subseteq \mathbf{G}$ be a set, $n, m \geqslant 1$ be positive integers. Then

$$
\left|A^{n+m}-\Delta(A)\right| \geqslant|A|^{m}\left|A^{n}-\Delta(A)\right|
$$

and

$$
\left|A^{n+m}+\Delta(A)\right| \geqslant|A|^{m} \max \left\{\left|A^{n}+\Delta(A)\right|,\left|A^{n}-\Delta(A)\right|\right\} .
$$

In particular,

$$
\left|A^{2}-\Delta(A)\right|=\sum_{s \in A-A}\left|A-A_{s}\right| \geqslant|A||A-A|,
$$

and

$$
\left|A^{2}+\Delta(A)\right|=\sum_{s \in A-A}\left|A+A_{s}\right| \geqslant|A| \max \{|A+A|,|A-A|\}
$$

Proof. In view of [28], Proposition 11 it remains to prove the second bound from (25) in the case $m=1$ only, namely, that $\left|A^{n+1}+\Delta(A)\right| \geqslant|A|\left|A^{n}-\Delta(A)\right|, n \geqslant 1$. But $\left(a_{1}+a, \ldots, a_{n}+a, a_{n+1}+a\right) \in A^{n+1}+\Delta(A)$ iff $a_{n+1} \in A_{s_{1}, \ldots, s_{n}}$, where $s_{j}=a_{j}-a_{n+1}$, $\left(s_{1}, \ldots, s_{n}\right) \in A^{n}-\Delta(A)$. Thus

$$
\left|A^{n+1}+\Delta(A)\right|=\sum_{\left(s_{1}, \ldots, s_{n}\right) \in A^{n}-\Delta(A)}\left|A+A_{s_{1}, \ldots, s_{n}}\right| \geqslant|A|\left|A^{n}-\Delta(A)\right|
$$

and the result follows.

We will use the Katz-Koester trick [18]

$$
A-A_{s} \subseteq(A-A)_{-s}, \quad A+A_{s} \subseteq(A+A)_{s},
$$

and its generalization (see e.g. [34])

$$
A-A_{\vec{x}} \subseteq(A-A)_{-\vec{x}}, \quad A+A_{\vec{x}} \subseteq(A+A)_{\vec{x}}
$$

very often. [31].

Finally, recall some results from [33]. We begin with an analog of a definition from 
Definition 11. Let $\alpha>1$ be a real number, $\beta, \gamma \in[0,1]$. A set $A \subseteq \mathbf{G}$ is called $(\alpha, \beta, \gamma)$-connected if for any $B \subseteq A,|B| \geqslant \beta|A|$ the following holds

$$
\mathrm{E}_{\alpha}(B) \geqslant \gamma\left(\frac{|B|}{|A|}\right)^{2 \alpha} \mathrm{E}_{\alpha}(A)
$$

Thus, a set from Theorem 2 is a $(2, \beta, \gamma)$-connected set with $\beta, \gamma \gg 1$. The Hölder inequality implies that if $\mathrm{E}_{\alpha}(A) \leqslant \gamma^{-1}|A|^{2 \alpha}|A-A|^{1-\alpha}$ then $A$ is $(\alpha, \beta, \gamma)$-connected for any $\beta$. As was proved in [31] that for $\alpha=2$ every set $A$ always contains large connected subset. For integers $\alpha>2$, see the Appendix.

Our first lemma from [33] (where some operators were used in the proof) provides a nontrivial lower bound for $\mathrm{E}_{s}(A), s \in[1,2]$ in terms of $\mathrm{E}(A)$.

Lemma 12. Let $A \subseteq \mathbf{G}$ be a set, and $\beta, \gamma \in[0,1]$. Suppose that $A$ is $(2, \beta, \gamma)$-connected with $\beta \leqslant 1 / 2$. Then for any $s \in[1,2]$ the following holds

$$
\mathrm{E}_{s}(A) \geqslant 2^{-5} \gamma|A|^{1-s / 2} \mathrm{E}^{s / 2}(A) .
$$

The second lemma from [33] provides an upper bound for eigenvalues of some operators. To avoid of using the operators notation we formulate the result in the following way.

Lemma 13. Let $A \subseteq \mathbf{G}$ be a set. Then for an arbitrary function $f: A \rightarrow \mathbb{C}$ one has

$$
\mathrm{E}(A, f) \leqslant \mathrm{E}_{3}^{1 / 2}(A)\|f\|_{2}^{2} .
$$

Further, there is a set $A^{\prime} \subseteq A,\left|A^{\prime}\right| \geqslant|A| / 2$, namely,

$$
A^{\prime}:=\left\{x:((A * A) \circ A)(x) \leqslant 2 \mathrm{E}(A)|A|^{-1}\right\}
$$

such that for any function $f: A^{\prime} \rightarrow \mathbb{C}$ the following holds

$$
\mathrm{E}(A, f) \leqslant \frac{2 \mathrm{E}(A)}{|A|} \cdot\|f\|_{2}^{2} .
$$

Moreover for any even real function $g$ there is a set $A^{\prime} \subseteq A,\left|A^{\prime}\right| \geqslant|A| / 2$ such that for any function $f: A^{\prime} \rightarrow \mathbb{C}$ one has

$$
\sum_{x} g(x)(\bar{f} \circ f)(x)=\sum_{x} g(x)(f \circ \bar{f})(x) \leqslant 2|A|^{-1} \sum_{x} g(x)(A \circ A)(x) \cdot\|f\|_{2}^{2} .
$$


Note that if $f=\tilde{A}, \tilde{A} \subseteq A$ then bound (31) can be obtained using the Cauchy-Schwarz inequality. Further, estimate (34) is a generalization of (33) which was proved in [33], see Lemma 44. Bound (34) can be obtained in a similar way.

We finish the section noting a generalization of formula (23) of Lemma 8. That is just a part of Lemma 4.2 from [33].

Lemma 14. Let $A, B \subseteq \mathbf{G}$ be finite sets, $S \subseteq \mathbf{G}$ be a set such that $A+B \subseteq S$. Suppose that $\psi$ is a function on $\mathbf{G}$. Then

$$
|B|^{2} \cdot\left(\sum_{x} \psi(x)(A \circ A)(x)\right)^{2} \leqslant \mathrm{E}_{3}(B, A) \sum_{x} \psi^{2}(x)(S \circ S)(x) .
$$

\section{Structural results}

In this section we obtain several general structural results, some of which have applications to sum-products phenomenon, for example. These results are closely related to the BalogSzemerédi-Gowers Theorem. We adopt the convention of writing $\mathbf{G}$ as an additive group. The proofs follow the arguments from [28] and [29].

Now we formulate the first result of the section.

Proposition 15. Let $A \subseteq \mathbf{G}$ be a finite set, and $M \geqslant 1, \eta \in(0,1]$ be real numbers. Let $\mathrm{E}(A)=|A|^{3} / K$. Suppose that for some set $P \subseteq A-A$ the following holds

$$
\sum_{s \in P}(A \circ A)(s)=\eta|A|^{2}
$$

and

$$
\sum_{s \in P}\left|A \pm A_{s}\right| \leqslant M K|A|^{2}
$$

Then for any $\varepsilon \in(0,1)$, there are two sets $H \subseteq \mathbf{G}, \Lambda \subseteq \mathbf{G}$ and $z \in \mathbf{G}$ such that

$$
\begin{gathered}
|(H+z) \cap A| \gg_{M, \eta^{-1}, K^{\varepsilon}} \frac{\mathrm{E}(A)}{|A|^{2}}, \quad|\Lambda| \ll_{M, \eta^{-1}, K^{\varepsilon}} \frac{|A|}{|H|}, \\
|H-H| \ll_{M, \eta^{-1}, K^{\varepsilon}}|H|,
\end{gathered}
$$

and

$$
|A \bigcap(H+\Lambda)| \gg_{M, \eta^{-1}, K^{\varepsilon}}|A| .
$$


Proof. Using Lemma 8 with $Q=P$, we see that

$$
\mathrm{E}_{3}(A) \geqslant \frac{\eta^{2}|A|^{4}}{M K}
$$

We have

$$
\sum_{s:\left|A_{s}\right|<\frac{|A| \eta^{2}}{2 K M}} \mathrm{E}\left(A, A_{s}\right) \leqslant\left(\frac{|A| \eta^{2}}{2 K M}\right) \sum_{s}\left|A_{s}\right||A|=\frac{|A|^{4} \eta^{2}}{2 K M} .
$$

Applying Lemma 7, that is the formula $\mathrm{E}_{3}(A)=\sum_{s} \mathrm{E}\left(A, A_{s}\right)$, combining it with (41), we get

$$
\sum_{s:\left|A_{s}\right| \geqslant 2^{-1} \eta^{2} M^{-1} K^{-1}|A|} \mathrm{E}\left(A, A_{s}\right) \geqslant \frac{\eta^{2}|A|^{4}}{2 M K} .
$$

Put

$$
\mu:=\max _{s:\left|A_{s}\right| \geqslant 2^{-1} \eta^{2} M^{-1} K^{-1}|A|} \frac{\mathrm{E}\left(A, A_{s}\right)}{|A|\left|A_{s}\right|^{2}} .
$$

Using (42), we have

$$
\mu|A| \mathrm{E}(A) \geqslant \mu|A| \cdot \sum_{s:\left|A_{s}\right| \geqslant 2^{-1} \eta^{2} M^{-1} K^{-1}|A|}\left|A_{s}\right|^{2} \geqslant \frac{\eta^{2}|A|^{4}}{2 M K} .
$$

Thus, $\mu \geqslant \frac{\eta^{2}}{2 M}$. Hence there is an $s$ with $\left|A_{s}\right| \geqslant 2^{-1} \eta^{2} M^{-1} K^{-1}|A|$ and such that $\mathrm{E}\left(A, A_{s}\right) \geqslant 2^{-1} M^{-1} \eta^{2}|A|\left|A_{s}\right|^{2}$. Applying the asymmetric version of Balog-SzemerédiGowers Theorem 6, we find two sets $\Lambda, H$ such that (38) - (40) take place. This completes the proof.

We write the fact that sets $A, H, \Lambda$ satisfy (38)-(40) with $\eta \gg 1$ as

$$
A \approx_{M, K^{\varepsilon}} \Lambda \dot{+} H
$$

Note that the degree of polynomial dependence in formula (43) is a function on $\varepsilon$.

Example 16. Let $H \subseteq \mathbf{F}_{2}^{n}$ be a subspace and $\Lambda \subseteq \mathbf{F}_{2}^{n}$ be a dissociated set (basis). Put $A=H \dot{+} \Lambda$, where $\dot{+}$ means the direct sum, and $|\Lambda|=K$. Detailed discussion of the example can be found, e.g. in [33]. If $s \in H$ then $A_{s}=A$ and hence $A+A_{s}=A+A$. If $s \in(A+A) \backslash H$ then $A_{s}$ is the disjoint union of two shifts of $H$ and thus $\left|A+A_{s}\right| \leqslant 2|A|$. Whence

$$
\sum_{s \in A+A}\left|A+A_{s}\right| \leqslant|H||A+A|+2|A+A||A| \ll K|A|^{2},
$$

and $\mathrm{E}(A) \sim|A|^{3} / K$. It means that condition (37) takes place in the case $A=H \dot{+} \Lambda$.

Taking $P=A-A$ and applying Proposition 15 as well as formulas (26), (27) of Lemma 10, we obtain the following consequence. 
Corollary 17. Let $A \subseteq \mathbf{G}$ be a set, $M \in \mathbb{R}, \varepsilon \in(0,1)$ and $\mathrm{E}(A)=|A|^{3} / K$. Then either

$$
\left|A^{2} \pm \Delta(A)\right| \geqslant M K|A|^{2}
$$

or $A \approx_{M, K^{\varepsilon}} \Lambda \dot{+} H$.

Note that for any set $A \subseteq \mathrm{G}$ with $\mathrm{E}(A)=|A|^{3} / K$ the inequality $\left|A^{2} \pm \Delta(A)\right| \geqslant K|A|^{2}$ follows from Lemma 8 and a trivial estimate $\mathrm{E}_{3}(A) \leqslant|A| \mathrm{E}(A)$. We will deal with the reverse condition $\mathrm{E}_{3}(A) \gg|A| \mathrm{E}(A)$ in Proposition 20 and Theorem 21 below.

The next corollary shows that if a set $A$ is not close to a set of the form $\Lambda \dot{+} H$ then there is some imbalance (in view of Plünnecke-Ruzsa inequality (18)) between doubling constant and additive energy of $A$ or $A-A$.

Corollary 18. Let $A \subseteq \mathbf{G}$ be a set, $M, \varepsilon \in(0,1)$ be real numbers and $|(A-A) \pm(A-A)| \gg$ $|A-A|^{3} /|A|^{2}$. Then either

$$
\mathrm{E}(A) \gg \frac{M^{1 / 2}|A|^{4}}{|A-A|} \quad \text { or } \quad \mathrm{E}(A-A) \gg \frac{M|A-A|^{4}}{|(A-A) \pm(A-A)|}
$$

or $A \approx_{M, K^{\varepsilon}} \Lambda+H$, where $K=|A-A| /|A|$.

Proof. Put $D=A-A$. Suppose that $\mathrm{E}(A) \ll \frac{M^{1 / 2}|A|^{4}}{|D|}$ because otherwise we are done. In view of Corollary 17 one can assume that $\sum_{s}\left|A-A_{s}\right| \geqslant M^{1 / 2}|A||D|$. Thus, by the Katz-Koester trick (28)

$$
A-A_{s} \subseteq(A-A)_{-s}, \quad A+A_{s} \subseteq(A+A)_{s},
$$

and the Cauchy-Schwarz inequality, we get

$$
|D| \mathrm{E}(D) \geqslant\left(\sum_{s \in D}(D \circ D)(s)\right)^{2} \geqslant\left(\sum_{s \in D}\left|A-A_{s}\right|\right)^{2} \geqslant\left(M^{1 / 2}|A||D|\right)^{2} .
$$

Hence

$$
\mathrm{E}(D) \geqslant M|D||A|^{2} \gg \frac{M|D|^{4}}{|(A-A) \pm(A-A)|}
$$

where the assumption of the corollary has been used. This completes the proof.

The quantities $\mathrm{E}(A \pm A)$ (and hence $\left|A^{2} \pm \Delta(A)\right|$ in view of Lemma 10, see also Proposition 29 below) appear in sum-products results (in multiplicative form). For example, in [21] the following theorem was proved.

Theorem 19. Let $A, B \subseteq \mathbb{R}$ be finite sets. Then

$$
|B+A A|^{3} \gg \frac{|B| \mathrm{E}^{\times}(A A)}{\log |A|} .
$$


Here $\mathrm{E}^{\times}(A):=\left|\left\{a_{1} a_{2}=a_{3} a_{4}: a_{1}, a_{2}, a_{3}, a_{4} \in A\right\}\right|$. Thus, by the obtained results, we have, roughly, that either $\mathrm{E}^{\times}(A), \mathrm{E}^{\times}(A A)$ can be estimated better then by Lemma 10 , see formulas (26), (27) or $A$ has the rigid structure $A \approx \Lambda \cdot H$. Usually, the last case is easy to deal with. Similar methods were used in [21].

Now we obtain another structural result. Using Lemma 8 as well as a trivial estimate $\mathrm{E}_{3}(A) \leqslant|A| \mathrm{E}(A)$ one can derive Proposition 15 from Proposition 20 below.

Proposition 20. Let $A \subseteq \mathbf{G}$ be a set, and $M \geqslant 1$ be a real number. Suppose that

$$
\mathrm{E}_{3}(A) \geqslant \frac{|A| \mathrm{E}(A)}{M}
$$

Then there is $A^{\prime} \subseteq A$ such that

$$
\left|A^{\prime}\right| \gg \frac{\mathrm{E}(A)}{|A|^{2}(M \log M)^{5}}
$$

and

$$
\left|A^{\prime}-A^{\prime}\right| \ll M^{15} \log ^{16} M \cdot\left|A^{\prime}\right| .
$$

Further, take any $\varepsilon \in(0,1)$ and put $K:=\frac{|A|^{3}}{\mathrm{E}(A)}$. Then $A \approx_{M, K^{\varepsilon}} \Lambda \dot{+} H$.

Proof. First of all prove (46), (47). Let

$$
P_{j}=\left\{x: 2^{j-1}|A| /\left(2^{2} M\right)<\left|A_{x}\right| \leqslant 2^{j}|A| /\left(2^{2} M\right)\right\}, \quad j \in[L],
$$

where $L=[\log (4 M)]$. By the pigeonhole principle there is $j \in[L]$ such that

$$
\frac{|A| \mathrm{E}(A)}{2 M L} \leqslant \frac{\mathrm{E}_{3}(A)}{2 L} \leqslant \sum_{x \in P_{j}}\left|A_{x}\right|^{3}
$$

Put $P=P_{j}$ and $\Delta=2^{j}|A| /\left(2^{2} M\right)$. Thus

$$
\frac{8 M \mathrm{E}(A)}{2^{2 j}|A| L} \leqslant \sum_{x} P(x)(A \circ A)(x)=\sum_{x} A(x)(A \circ P)(x) .
$$

Hence, by the Cauchy-Schwarz inequality

$$
\frac{2^{6} M^{2} \mathrm{E}^{2}(A)}{2^{4 j}|A|^{3} L^{2}} \leqslant \mathrm{E}(A, P) \leqslant(\mathrm{E}(A))^{1 / 2}(\mathrm{E}(P))^{1 / 2} .
$$

Note that

$$
\mathrm{E}(A) \geqslant \sum_{x \in P}\left|A_{x}\right|^{2} \geqslant \frac{|P||A|^{2} 2^{2 j-2}}{2^{4} M^{2}}
$$

and therefore

$$
|P| \leqslant 2^{6} 2^{-2 j} M^{2} \mathrm{E}(A)|A|^{-2} .
$$


It follows that

$$
\mathrm{E}(P) \geqslant \frac{2^{12} M^{4}(\mathrm{E}(A))^{3}}{2^{8 j}|A|^{6} L^{4}} \geqslant \frac{|P|^{3}}{2^{6} M^{2} 2^{2 j} L^{4}} \geqslant \frac{|P|^{3}}{2^{10} M^{4} L^{4}}:=\mu|P|^{3} .
$$

By Theorem 5 there is $P^{\prime} \subseteq P$ such that $\left|P^{\prime}\right| \gg \mu|P|$ and $\left|P^{\prime}-P^{\prime}\right| \ll \mu^{-4}\left|P^{\prime}\right|$. Note that

$$
\sum_{x \in A}\left(A \circ P^{\prime}\right)(x)=\sum_{x \in P^{\prime}}(A \circ A)(x) \geqslant \frac{\left|P^{\prime}\right| 2^{j-3}|A|}{M},
$$

and so there exists $x \in A$ such that the set $A^{\prime}:=A \cap\left(P^{\prime}+x\right)$ has the size at least $\left|P^{\prime}\right| 2^{j-3} M^{-1}$. We have

$$
\left|A^{\prime}-A^{\prime}\right| \leqslant\left|P^{\prime}-P^{\prime}\right| \ll \mu^{-4}\left|P^{\prime}\right| \ll \mu^{-4} 2^{-j} M\left|A^{\prime}\right| \ll M^{15} L^{16}\left|A^{\prime}\right| .
$$

Finally, from (48), say, one has

$$
|P| \gg \frac{M^{2} \mathrm{E}(A)}{2^{3 j}|A|^{2} L} \gg \frac{\mathrm{E}(A)}{M|A|^{2} L}
$$

and because

$$
\left|A^{\prime}\right| \geqslant\left|P^{\prime}\right| 2^{j-3} M^{-1} \gg \mu|P| \cdot 2^{j-3} M^{-1}
$$

the result follows.

To obtain (38)-(40), that is $A \approx_{M, K^{\varepsilon}} \Lambda \dot{+} H, K=|A|^{3} \mathrm{E}^{-1}(A)$, note that by the first inequality of (49) and the bound $|P| \leqslant 2^{6} 2^{-2 j} M^{2} \mathrm{E}(A)|A|^{-2}$, we have

$$
\mathrm{E}(A, P) \geqslant \frac{2^{6} M^{2} \mathrm{E}^{2}(A)}{2^{4 j}|A|^{3} L^{2}} \geqslant \frac{|A||P|^{2}}{2^{6} L^{2} M^{2}} .
$$

Also, by the definition of the number $K$, and inequality (53) the following holds

$$
|A| /|P| \ll M L K \ll_{M} K .
$$

Applying the asymmetric version of Balog-Szemerédi-Gowers Theorem 6 with $A=A$, $B=P$, and recalling (53), we obtain the required inequalities, excepting the first inequality of (38), where it remains to replace $P$ by $A$. Put $H^{\prime}=(H+z) \cap P$. We have $\left|H^{\prime}\right| \gg_{M, K^{\varepsilon}}|P|$. Thus, by the definition of the number $\Delta$ and estimate (53), we obtain

$$
\sum_{x \in A}\left(A \circ H^{\prime}\right)(x)=\sum_{x \in H^{\prime}}(A \circ A)(x) \geqslant 2^{-1} \Delta\left|H^{\prime}\right| \gg_{M, K^{\varepsilon}} \Delta|P| \gg_{M, K^{\varepsilon}} \frac{\mathrm{E}(A)}{|A|} .
$$

Hence there is $w \in A$ such that

$$
|(H+w) \cap A| \geqslant\left|\left(H^{\prime}+w\right) \cap A\right| \gg_{M, K^{\varepsilon}} \frac{\mathrm{E}(A)}{|A|^{2}} .
$$

This completes the proof. 
Assumption (45) of the Proposition 20 is a generalisation of the usual condition $\mathrm{E}(A) \geqslant$ $\frac{|A|^{3}}{M}$ (because $\mathrm{E}(A)|A|^{3} M^{-1} \leqslant \mathrm{E}^{2}(A) \leqslant \mathrm{E}_{3}(A)|A|^{2}$ ) and $\mathrm{E}_{3}(A) \geqslant \frac{|A|^{4}}{M}$ (because $\mathrm{E}_{3}(A) \geqslant$ $\left.|A|^{4} M^{-1} \geqslant|A| \mathrm{E}(A) M^{-1}\right)$. Further, one can check that the same consequences (38)-(40) hold if we replace condition (45) by $\mathrm{E}_{s}(A) \geqslant|A| \mathrm{E}_{s-1}(A) / M, s \geqslant 3$. Let us write the correspondent result.

Theorem 21. Let $A \subseteq \mathbf{G}$ be a set, $s \geqslant 3$ be a positive integer, and $M \geqslant 1$ be a real number. Suppose that

$$
\mathrm{E}_{s}(A) \geqslant \frac{|A| \mathrm{E}_{s-1}(A)}{M} .
$$

Take any $\varepsilon \in(0,1)$ and put $K:=\frac{|A|^{s}}{\mathrm{E}_{s-1}(A)}$. Then $A \approx_{s, M, K^{\varepsilon}} \Lambda \dot{+} H,|H| \gg_{s, M, K^{\varepsilon}}|A| / K$.

Proof. The arguments almost repeat the proof of Proposition 20, so we skip some details. Using dyadic pigeonholing and the assumption, we find $P \subseteq A-A, \Delta<\left|A_{x}\right| \leqslant 2 \Delta$, $x \in P$ with

$$
M^{-1}|A| \mathrm{E}_{s-1}(A) \leqslant \mathrm{E}_{s}(A) \ll_{\log M} \sum_{x \in P}\left|A_{x}\right|^{s} \ll_{\log M} \Delta^{s-1} \sigma_{P}(A) .
$$

Thus, by the Cauchy-Schwarz inequality

$$
M^{-2}|A| \mathrm{E}_{s-1}^{2}(A) \Delta^{-2(s-1)} \ll_{\log M} \mathrm{E}(A, P) .
$$

On the other hand $|P| \Delta^{s-1} \leqslant \mathrm{E}_{s-1}(A)$ and hence

$$
\mathrm{E}(A, P) \gg_{\log M} M^{-2}|A||P|^{2} .
$$

Note that by (56) and our choice of the set $P$, we have

$$
\frac{|A|}{|P|} \ll_{M} \frac{\Delta^{s}}{\mathrm{E}_{s-1}(A)} \leqslant \frac{|A|^{s}}{\mathrm{E}_{s-1}(A)},
$$

and, again,

$$
|P| \sim_{\log M} \mathrm{E}_{s}(A) \Delta^{-s} \geqslant M^{-1}|A| \mathrm{E}_{s-1}(A) \Delta^{-s}=\frac{|A|^{s+1}}{M K \Delta^{s}} \geqslant \frac{|A|}{M K} .
$$

After that apply the asymmetric version of Balog-Szemerédi-Gowers Theorem 6 and an analog of the arguments from (54) - (55). This concludes the proof.

The more general assumption $\mathrm{E}_{s}(A) \geqslant|A|^{k} \mathrm{E}_{s-k}(A) / M^{k}$ implies that for some $j \in[k]$ one has $\mathrm{E}_{s-j+1}(A) \geqslant|A| \mathrm{E}_{s-j}(A) / M$. Thus, we have considered the common case. Note, finally, that estimates (46), (47) are the best possible. Indeed, take $\mathbf{G}=\mathbf{F}_{2}^{n}, A=H \dot{+} \Lambda$, where $H \leqslant \mathbf{F}_{2}^{n}$ is a linear subspace and $\Lambda$ is a dissociated set (basis).

Now we can prove a criterium for sets having critical relation between $\mathrm{E}(A)$ and $\mathrm{E}_{3}(A)$ energies. 
Theorem 22. Let $A \subseteq \mathbf{G}$ be a set, and $M \geqslant 1, \varepsilon \in(0,1)$ be real numbers. Put $K:=\frac{|A|^{3}}{\mathrm{E}(A)}$. Then

$$
\mathrm{E}_{3}(A) \gg_{M, K^{\varepsilon}}|A| \mathrm{E}(A)
$$

iff

$$
A \approx_{M, K^{\varepsilon}} \Lambda+H .
$$

Proof. In view of Proposition 20 it remains to prove that if $A \approx_{M, K^{\varepsilon}} \Lambda \dot{+} H$ then $\mathrm{E}_{3}(A) \gg_{M, K^{\varepsilon}}|A| \mathrm{E}(A)$. Put $A_{1}=A \cap(H+\Lambda)$. We have $\left|A_{1}\right| \gg_{M, K^{\varepsilon}}|A|$. Then $|A|^{2}|H|^{2} \ll_{M, K^{\varepsilon}}\left|A_{1}\right|^{2}|H|^{2} \leqslant \mathrm{E}\left(A_{1}, H\right)\left|A_{1}-H\right| \leqslant \mathrm{E}(A, H)|\Lambda||H-H| \ll_{M, K^{\varepsilon}} \mathrm{E}(A, H)|A|$. Thus

$$
\left(|A||H|^{2}\right)^{3} \ll_{M, K^{\varepsilon}}\left(\sum_{x}(A \circ A)(x)(H \circ H)(x)\right)^{3} \leqslant \mathrm{E}_{3}(A) \mathrm{E}_{3 / 2}^{2}(H) \leqslant \mathrm{E}_{3}(A)|H|^{5} .
$$

In other words

$$
\mathrm{E}_{3}(A) \gg_{M, K^{\varepsilon}}|A|^{3}|H| \gg_{M, K^{\varepsilon}} \mathrm{E}(A)|A| .
$$

To get the last estimate we have used the fact $|H| \gg_{M, K^{\varepsilon}} \mathrm{E}(A)|A|^{-2}$. This completes the proof.

Recall that

$$
\mathrm{T}_{k}(A):=\left|\left\{a_{1}+\cdots+a_{k}=a_{1}^{\prime}+\cdots+a_{k}^{\prime}: a_{1}, \ldots, a_{k}, a_{1}^{\prime}, \ldots, a_{k}^{\prime} \in A\right\}\right| .
$$

We conclude the section proving a "dual" analogue of Proposition 20, that is we replace the condition on $\mathrm{E}_{3}(A)$ with a similar condition for $\mathrm{T}_{4}(A)$ and moreover for $\mathrm{T}_{s}(A)$. Again, the proof follows the arguments from [28].

Theorem 23. Let $A \subseteq \mathbf{G}$ be a set, and $M \geqslant 1$ be a real number. Suppose that

$$
\mathrm{T}_{4}(A) \geqslant \frac{|A|^{4} \mathrm{E}(A)}{M} .
$$

Then there is $A^{\prime} \subseteq A$ such that

$$
\left|A^{\prime}\right| \gg \frac{|A|}{M^{3} \log ^{\frac{16}{3}}|A|}
$$

and

$$
\left|n A^{\prime}-m A^{\prime}\right| \ll\left(M^{3} \log ^{4}|A|\right)^{4(n+m)} M\left|A^{\prime}\right| \cdot \frac{|A|^{3}}{\mathrm{E}(A)}
$$

for every $n, m \in \mathbb{N}$.

Moreover, if

$$
\mathrm{T}_{2 s}(A) \geqslant \frac{|A|^{2 s} \mathrm{~T}_{s}(A)}{M},
$$

$s \geqslant 2$ then formulas (58), (59) take place. Conversely, bounds (58), (59) imply that $\mathrm{T}_{2 s}(A) \gg_{M, \log |A|, s}|A|^{2 s} \mathrm{~T}_{s}(A)$. 
Proof. Put $\mathrm{T}_{s}=\mathrm{T}_{s}(A), \mathrm{T}_{2 s}=\mathrm{T}_{2 s}(A), a=|A|, L_{s}=\left[\log \left(16 M a^{2 s-1} / \mathrm{T}_{s}\right)\right] \ll_{s} \log a$. Let

$$
P_{j}=\left\{x: 2^{j-1} \mathrm{~T}_{s} /\left(2^{4} M a^{s}\right)<\left(A *_{s-1} A\right)(x) \leqslant 2^{j} \mathrm{~T}_{s} /\left(2^{4} M a^{s}\right)\right\}, \quad j \in\left[L_{s}\right] .
$$

Put $f_{j}(x)=P_{j}(x)\left(A *_{s-1} A\right)(x)$. Thus,

$$
\left(A *_{s-1} A\right)(x)=\sum_{j=1}^{L_{s}} f_{j}(x)+\Omega(x)\left(A *_{s-1} A\right)(x),
$$

where $\Omega=\left\{x \quad:\left(A *_{s-1} A\right)(x) \leqslant 2^{-4} M^{-1} \mathrm{~T}_{s} a^{-s}\right\}$. Substituting formula (61) into the identity

$$
\mathrm{T}_{2 s}(A)=\sum_{x}\left(\left(A *_{s-1} A\right) \circ\left(A *_{s-1} A\right)\right)^{2}(x)
$$

and using assumptions (57), (60), combining with the definition of sets $P_{j}, \Omega$, we have

$$
2^{-1} \mathrm{~T}_{2 s}(A) \leqslant \sum_{j_{1}, j_{2}, j_{3}, j_{4}=1}^{L_{s}} \sum_{x}\left(f_{j_{1}} \circ f_{j_{2}}\right)(x)\left(f_{j_{3}} \circ f_{j_{4}}\right)(x) .
$$

Applying the Hölder inequality, we get

$$
2^{-1} L_{s}^{-3} \mathrm{~T}_{2 s}(A) \leqslant \sum_{j=1}^{L_{s}} \sum_{x}\left(f_{j} \circ f_{j}\right)(x)\left(f_{j} \circ f_{j}\right)(x) .
$$

By the pigeonhole principle there is $j \in\left[L_{s}\right]$ such that

$$
\frac{a^{2 s} \mathrm{~T}_{s}}{2 M L_{s}^{4}} \leqslant \frac{\mathrm{T}_{2 s}}{2 L_{s}^{4}} \leqslant \sum_{x}\left(f_{j} \circ f_{j}\right)(x)\left(f_{j} \circ f_{j}\right)(x) .
$$

Put $P=P_{j}, f=f_{j}$ and $\Delta=2^{j} \mathrm{~T}_{s} /\left(2^{4} M a^{s}\right)$. Thus

$$
\frac{a^{2 s} \mathrm{~T}_{s}}{2 M L_{s}^{4} \Delta^{4}} \leqslant \mathrm{E}(P)
$$

Clearly, $|P| \leqslant 4 \mathrm{~T}_{s} \Delta^{-2}$. Using the last inequality, the definition of the number $\Delta$ and bound (63), we obtain

$$
\mathrm{E}(P) \geqslant \frac{a^{2 s} \mathrm{\top}_{s}}{2 M L_{s}^{4} \Delta^{4}} \geqslant|P|^{3} \frac{a^{2 s} \Delta^{2}}{2^{7} M L_{s}^{4} \mathrm{~T}_{s}^{2}} \geqslant|P|^{3} \frac{2^{2 j}}{2^{15} M^{3} L_{s}^{4}} \geqslant \frac{|P|^{3}}{2^{15} M^{3} L_{s}^{4}}:=\mu|P|^{3} .
$$

To estimate the size of $P$ we note by (63) that

$$
|P|^{3} \geqslant \frac{a^{2 s} \mathrm{~T}_{s}}{2 M L_{s}^{4} \Delta^{4}}
$$


After that use arguments (51) - (52) of the proof of Proposition 20. By Theorem 5 there is $P^{\prime} \subseteq P$ such that $\left|P^{\prime}\right| \gg \mu|P|$ and $\left|P^{\prime}-P^{\prime}\right| \ll \mu^{-4}\left|P^{\prime}\right|$. Applying Plünnecke-Ruzsa inequality (18), we obtain

$$
\left|n P^{\prime}-m P^{\prime}\right| \ll \mu^{-4(n+m)}\left|P^{\prime}\right|
$$

for every $n, m \in \mathbb{N}$. We have

$$
\Delta\left|P^{\prime}\right| \leqslant \sum_{x}\left(A *_{s-1} A\right)(x) P^{\prime}(x)=\sum_{x_{1}, \ldots, x_{s-1} \in A}\left(A \circ P^{\prime}\right)\left(x_{1}+\cdots+x_{s-1}\right) .
$$

By (64) and the definition of the number $\Delta$ there is $x \in(s-1) A$ such that the set $A^{\prime}:=A \cap\left(P^{\prime}-x\right)$ has the size at least

$$
\left|A^{\prime}\right| \gg\left|P^{\prime}\right| \Delta a^{-(s-1)} \geqslant \mu|P| \Delta a^{-(s-1)} \gg \mu\left(\frac{\mathrm{T}_{s}}{M L_{s}^{4} \Delta a^{s-3}}\right)^{1 / 3} \gg \frac{2^{5 j / 3} a}{M^{3} L_{s}^{16 / 3}} \gg \frac{a}{M^{3} L_{s}^{16 / 3}} .
$$

We have by (65) that

$$
\begin{gathered}
\left|n A^{\prime}-m A^{\prime}\right| \leqslant\left|n P^{\prime}-m P^{\prime}\right| \ll \mu^{-4(n+m)}\left|P^{\prime}\right| \ll \mu^{-4(n+m)}\left|A^{\prime}\right| a^{s-1} \Delta^{-1} \ll \\
\ll \mu^{-4(n+m)} M\left|A^{\prime}\right| \cdot \frac{a^{2 s-1}}{\mathrm{~T}_{s}}
\end{gathered}
$$

for every $n, m \in \mathbb{N}$.

Conversely, applying bound (59) with $n=m=s$, combining with the Cauchy-Schwarz inequality, we obtain

$$
\begin{gathered}
\left|A^{\prime}\right|^{4 s} \leqslant\left(\sum_{x}\left(\left(A^{\prime} *_{s-1} A^{\prime}\right) \circ\left(A^{\prime} *_{s-1} A^{\prime}\right)\right)(x)\right)^{2} \leqslant \mathrm{~T}_{2 s}\left(A^{\prime}\right)\left|s A^{\prime}-s A^{\prime}\right| \ll_{s} \\
\ll_{s}\left(M^{3} \log ^{4}|A|\right)^{8 s} M \cdot\left|A^{\prime}\right| \frac{|A|^{2 s-1}}{\mathrm{~T}_{s}(A)} \mathrm{T}_{2 s}(A) .
\end{gathered}
$$

Using (58), we get

$$
\begin{gathered}
\mathrm{T}_{2 s}(A) \gg_{s} \mathrm{~T}_{s}(A)\left|A^{\prime}\right|^{4 s-1}|A|^{-(2 s-1)}\left(M^{3} \log ^{4}|A|\right)^{-8 s} M^{-1} \gg_{s} \\
\gg_{s} \mathrm{~T}_{s}(A)|A|^{2 s} M^{-36 s+2} \log ^{-54 s}|A| .
\end{gathered}
$$

In other words, $\mathrm{T}_{2 s}(A) \gg_{M, \log |A|, s}|A|^{2 s} \mathrm{~T}_{s}(A)$. This completes the proof.

So, we have proved in Theorem 23 that, roughly speaking, $A^{\prime}-A^{\prime}$ is a set with small (in terms of the parameter $M$ ) doubling and vice versa. Thus, $A^{\prime}$ does not equal to a set with small doubling but $A^{\prime}-A^{\prime}$ does. Results of such a sort were obtained in [28], [32] and [33]. Note, that we need in multiple $|A|^{3} \mathrm{E}^{-1}(A)$ in (59), because by (58) and the Cauchy-Schwarz inequality, we have the same lower bound for $\left|A^{\prime}-A^{\prime}\right|$.

It is easy to see that an analog of Theorem 23 takes place if one replace (57) onto condition $\mathrm{T}_{s}(A) \geqslant|A|^{2(s-2)} \mathrm{E}(A) / M$, where $s$ is an even number, $s \geqslant 4$, and, further, even more general relations between $\mathrm{T}_{k}$ energies can be reduced to the last case (and Theorem 23) via a trivial estimate $\mathrm{T}_{s}(A) \leqslant|A|^{2} \mathrm{~T}_{s-1}(A)$. We do not need in such generalizations in the paper. 


\section{Sumsets: preliminaries}

Let $A \subseteq \mathrm{G}$ be a set. Before studying the energies of sumsets or difference sets we concentrate on a following related question, which was asked to the author by Tomasz Schoen. Namely, what can be proved nontrivial concerning lower bounds for $\left|A \pm A_{s}\right|$, $s \neq 0$ ? The connection with $A \pm A$ is obvious in view of Katz-Koester trick (28). We start with a result in the direction.

Theorem 24. Let $A \subseteq \mathbf{G}$ be a set. If $\mathrm{E}_{3}(A) \geqslant 2|A|^{3}$ then

$$
\left(\max _{s \neq 0}\left|A \pm A_{s}\right|\right)^{3} \gg \frac{|A|^{10}}{|A-A| \mathrm{E}^{2}(A)} .
$$

Now suppose that $A$ is $(3, \beta, \gamma)$-connected with $\beta \leqslant 1 / 2$, and $\mathrm{E}_{3}(A) \geqslant 2^{4} \gamma^{-1}|A|^{3}$. Then

$$
\left(\max _{s \neq 0}\left|A \pm A_{s}\right|\right)^{2} \gg \gamma \frac{|A|^{5}}{\mathrm{E}(A)} .
$$

Proof. Write $\mathrm{E}=\mathrm{E}(A), \mathrm{E}_{3}=\mathrm{E}_{3}(A)$, and $a=|A|$. Let us begin with (66). Denote by $\omega$ the maximum in (66). By the Cauchy-Schwarz inequality and formula (31) of Lemma 13, we obtain

$$
a^{2}\left|A_{s}\right|^{2} \leqslant \mathrm{E}\left(A, A_{s}\right)\left|A \pm A_{s}\right| \leqslant \mathrm{E}_{3}^{1 / 2}\left|A_{s}\right|\left|A \pm A_{s}\right| .
$$

Multiplying the last inequality by $\left|A_{s}\right|$, summing over $s \neq 0$ and using the assumption $\mathrm{E}_{3}(A) \geqslant 2|A|^{3}$, we get

$$
a^{2} \mathrm{E}_{3}^{1 / 2} \ll \omega \mathrm{E} .
$$

On the other hand, by Lemma 8, we have

$$
a^{6} \ll \mathrm{E}_{3} \sum_{s \in A-A, s \neq 0}\left|A \pm A_{s}\right| \leqslant \mathrm{E}_{3}|A-A| \omega .
$$

Combining (69) with (70), we obtain

$$
\omega^{3} \gg \frac{a^{10}}{|A-A| \mathrm{E}^{2}}
$$

as required.

Now let us obtain (67). Using Lemma 13, we find $A^{\prime},\left|A^{\prime}\right| \geqslant|A| / 2$ such that estimate (33) takes place. As in (68), we get

$$
a^{2}\left|A_{s}^{\prime}\right|^{2} \leqslant \mathrm{E}\left(A, A_{s}^{\prime}\right)\left|A \pm A_{s}^{\prime}\right| \leqslant \frac{2 \mathrm{E}}{a}\left|A_{s}^{\prime}\right|\left|A \pm A_{s}\right| .
$$

By assumption $\mathrm{E}_{3}(A) \geqslant 2^{4} \gamma^{-1}|A|^{3}$. Using the connectedness, we obtain

$$
\mathrm{E}_{3}\left(A^{\prime}\right) \geqslant \gamma \frac{\left|A^{\prime}\right|^{6}}{|A|^{6}} \mathrm{E}_{3}(A) \geqslant 2\left|A^{\prime}\right|^{3} .
$$


Multiplying inequality (71) by $\left|A_{s}^{\prime}\right|$, summing over $s \neq 0$, we have in view of $(72)$ that

$$
a^{2} \mathrm{E}_{3}\left(A^{\prime}\right) \ll a^{-1} \mathrm{EE}^{*}\left(A^{\prime}\right) \omega .
$$

Combining the last formula with first inequality from (72), we get

$$
a^{2} \gamma \mathrm{E}_{3}(A) \ll a^{-1} \mathrm{EE}^{*}\left(A^{\prime}\right) \omega .
$$

On the other hand, summing the first estimate from (71) over $s \neq 0$ and applying Lemma 7 , we see that

$$
a^{2} \mathrm{E}^{*}\left(A^{\prime}\right) \leqslant \omega \mathrm{E}_{3} .
$$

Combining (74), (75), we obtain

$$
\omega^{2} \gg \gamma \frac{a^{5}}{\mathrm{E}}
$$

This completes the proof.

From (66) it follows that if $|A-A|=K|A|, \mathrm{E}(A) \ll|A|^{3} / K, \mathrm{E}_{3}(A) \geqslant 2|A|^{3}$ then there is $s \neq 0$ such that $\left|A-A_{s}\right| \gg K^{1 / 3}|A|$ as well as there exists $s \neq 0$ with $\left|A+A_{s}\right| \gg K^{1 / 3}|A|$. It improves a trivial lower bound $\left|A \pm A_{s}\right| \geqslant|A|$. Using bound (67) one can show that there is $s \neq 0$ such that $\left|A-A_{s}\right| \gg K^{1 / 2}|A|$ (here $\mathrm{E}(A) \ll|A|^{3} / K$ ), provided by some connectedness assumptions take place.

We need in lower bounds on $\mathrm{E}_{k}(A)$ in Theorem 24 and Proposition 25 below to be separated from a very natural simple example, which can be called a "random sumset" case. Namely, take a random $A \subseteq \mathbf{G}$ and consider $A \pm A$. This "random sumset" has almost no structure (provided by $A \pm A$ is not a whole group, of course) and we cannot say something useful in the situation. It does not contradict Theorem 24 and Proposition 25 (see also the results of the next section) because the energies $\mathrm{E}_{k}(A)$ are really small in the case.

Now we give another proof of estimate (67) which can be derived from inequality (77), case $k=2$ below. Actually, it gives us even stronger inequality, namely, $\left|A^{2}-\Delta\left(A_{s}\right)\right| \gg$ $|A|^{5} \mathrm{E}^{-1}(A)$ for some $s \neq 0$, or, more generally, (see formulas (79), (80) below)

$$
\max _{s \neq 0}\left|A^{k} \pm \Delta\left(A_{s}\right)\right| \gg \max _{r \geqslant 1}\left\{\frac{|A|^{2 k+1} \mathrm{E}_{r+1}(A)}{\mathrm{E}_{r}(A) \mathrm{E}_{k+1}(A)}\right\},
$$

provided by some connectedness assumptions take place. In particular, taking $r=k$ and $r=1$ in the previous formula, we get

$$
\max _{s \neq 0}\left|A^{k} \pm \Delta\left(A_{s}\right)\right| \gg \max \left\{\frac{|A|^{2 k+1}}{\mathrm{E}_{k}(A)}, \frac{|A|^{2 k-1} \mathrm{E}(A)}{\mathrm{E}_{k+1}(A)}\right\} .
$$


Proposition 25. Let $A \subseteq \mathbf{G}$ be a set, $k \geqslant 2$ be a positive integer. Take two sets $D, S$ such that $A-A \subseteq D, A+A \subseteq S$. Then

$$
\begin{aligned}
& \gamma|A|^{2 k+1} \ll_{k} \sum_{x \neq 0}(A \circ A)^{k}(x) d_{k}(x) \leqslant \sum_{x \neq 0}(A \circ A)^{k}(x)(D \circ D)^{k}(x), \\
& \gamma|A|^{2 k+1} \ll_{k} \sum_{x \neq 0}(A \circ A)^{k}(x) s_{k}(x) \leqslant \sum_{x \neq 0}(A \circ A)^{k}(x)(S \circ S)^{k}(x),
\end{aligned}
$$

provided by $A$ is $(k+1, \beta, \gamma)$-connected with $\beta \leqslant 1 / 2$ and $\mathrm{E}_{k+1}(A) \geqslant 2^{k+2} \gamma^{-1}|A|^{k+1}$. Here $d_{k}(x)=\sum_{\alpha} D_{x}(\alpha)\left(D \circ D_{x}\right)^{k-1}(\alpha), s_{k}(x)=\sum_{\alpha} S_{x}(\alpha)\left(S_{x} * D\right)^{k-1}(\alpha)$.

Proof. Using Lemma 13, we find $A^{\prime},\left|A^{\prime}\right| \geqslant|A| / 2$ such that estimate (34) takes place with $g(z)=(A \circ A)^{k}(z)$. It follows that

$$
|A|^{2 k}\left|A_{x}^{\prime}\right|^{2} \leqslant\left|A^{k} \pm \Delta\left(A_{x}^{\prime}\right)\right| \mathrm{E}_{k+1}\left(A_{x}^{\prime}, A\right) \leqslant\left|A^{k} \pm \Delta\left(A_{x}\right)\right| \frac{2 \mathrm{E}_{k+1}(A)}{|A|}\left|A_{x}\right| .
$$

Multiplying the last inequality by $\left|A_{x}^{\prime}\right|^{k-1}$ and summing over $x \neq 0$ (to obtain (76) multiply by $\left.\left|A_{x}^{\prime}\right|^{r-1}\right)$, we get

$$
\gamma|A|^{2 k+1} \mathrm{E}_{k+1}(A) \ll|A|^{2 k+1} \mathrm{E}_{k+1}^{*}\left(A^{\prime}\right) \ll \mathrm{E}_{k+1}(A) \sum_{x \neq 0}\left|A^{k} \pm \Delta\left(A_{x}\right)\right|\left|A_{x}\right|^{k} .
$$

Here we have used the fact

$$
\mathrm{E}_{k+1}\left(A^{\prime}\right) \geqslant \gamma \frac{\left|A^{\prime}\right|^{2(k+1)}}{|A|^{2(k+1)}} \mathrm{E}_{k+1}(A) \geqslant 2\left|A^{\prime}\right|^{k+1}
$$

and the assumption $\mathrm{E}_{k+1}(A) \geqslant 2^{k+2} \gamma^{-1}|A|^{k+1}$. Note that by Katz-Koester trick (29), one has $\left|A^{k}-\Delta\left(A_{x}\right)\right| \leqslant d_{k}(x),\left|A^{k}+\Delta\left(A_{x}\right)\right| \leqslant s_{k}(x)$ (or just see the proof of Proposition 29 below). Finally, $d_{k}(x) \leqslant\left|D_{x}\right|^{k}, s_{k}(x) \leqslant\left|S_{x}\right|^{k}$ and we obtain (77). This completes the proof.

Using Katz-Koester trick or just the Cauchy-Schwarz inequality one can show that (77) trivially takes place in the case $k=1$ without any conditions on connectedness of $A$ or lower bounds for any sort of energy. Under the assumptions of Proposition 25 from (77) it follows that

$$
\gamma^{2}|A|^{4 k+2} \ll_{k} \mathrm{E}_{2 k}^{*}(A) \mathrm{E}_{2 k}^{*}(D)
$$

and similarly for $S$. Some weaker results but without any conditions on $A$ were obtained in $[28]$.

Results above give an interesting corollary on non-random sumsets/difference sets. Put $D=A-A, S=A+A$. Then for an arbitrary positive integer $k$ and any elements $a_{1}, \ldots, a_{k} \in A$, we have

$$
A \subseteq\left(D+a_{1}\right) \bigcap\left(D+a_{2}\right) \cdots \bigcap\left(D+a_{k}\right), \quad A \subseteq\left(S-a_{1}\right) \bigcap\left(S-a_{2}\right) \cdots \bigcap\left(S-a_{k}\right) .
$$


In particular, there is $x \in D, x \neq 0$ such that $\left|D_{x}\right|,\left|S_{x}\right| \geqslant|A|$ (also it follows from Katz-Koester inclusion (29)). By Corollary 17 (see also Lemma 10) one can improve it to $\left|D_{x}\right|,\left|S_{x}\right| \geqslant K^{\varepsilon}|A|$, where $K=|A|^{3} \mathrm{E}^{-1}(A)$. Theorem 24 gives us even stronger result.

Corollary 26. Let $A \subseteq \mathbf{G}$ be a set, $D=A-A, S=A+A, \mathrm{E}(A)=|A|^{3} / K$, Suppose that $A$ is $(3, \beta, \gamma)$-connected, $\beta \leqslant 1 / 2$, and $\mathrm{E}_{3}(A) \geqslant \gamma^{-1} 2^{4}|A|^{3}$. Then there is $x \neq 0$ such that $\left|D_{x}\right|,\left|S_{x}\right| \gg \gamma^{1 / 2} K^{1 / 2}|A|$.

One can get an analog of Corollary 26 for multiple intersections (81) but another types of energies will require in the case. Nevertheless, some weaker inequality of the form $\left|D_{\vec{x}}\right| \geqslant K^{\varepsilon}|A|$ can be obtained, using Proposition 20 and Theorem 21. Here $K=$ $|A|^{k+1} \mathrm{E}_{k}^{-1}(A), k \geqslant 2$. Interestingly, we do not even need in any connectedness in this weaker result.

Proposition 27. Let $A \subseteq \mathbf{G}$ be a set, $k \geqslant 2$ be a positive integer, $c \in(0,1]$ be a real number, $\mathrm{E}_{k}(A) \geqslant k^{2} \mathrm{E}_{k-1}(A)$, and $K=|A|^{k+1} \mathrm{E}_{k}^{-1}(A) \leqslant|A|^{1-c}, K_{1}=|A|^{3} \mathrm{E}^{-1}(A)$. Then for all sufficiently small $\varepsilon=\varepsilon(c)>0$ there is $\vec{x}=\left(x_{1}, \ldots, x_{k-1}\right)$ with distinct $x_{j}, j \in[k]$ such that

$$
\left|D_{\vec{x}}\right|,\left|S_{\vec{x}}\right| \gg_{k}|A| \cdot \min \left\{K^{\varepsilon}, c_{K^{\varepsilon}} K_{1}\right\}
$$

where the constant $c_{K^{\varepsilon}}$ satisfies $c_{K^{\varepsilon}} \gg_{K^{\varepsilon}} 1$ and, again, the degree of the polynomial dependence is a function on $c$.

Proof. Suppose not. Take any set $\mathcal{P} \subseteq A^{k-1}-\Delta(A)$. Applying Lemma 7, one has

$$
\begin{aligned}
|A|^{2}\left(\sum_{\vec{x} \in \mathcal{P}}\left|A_{\vec{x}}\right|\right)^{2}= & \left(\sum_{\vec{x} \in \mathcal{P}} \sum_{z}\left(A \circ A_{\vec{x}}\right)(z)\right)^{2} \leqslant \mathrm{E}_{k+1}(A) \sum_{\vec{x} \in \mathcal{P}}\left|A \pm A_{\vec{x}}\right| \leqslant \\
& \leqslant \mathrm{E}_{k+1}(A)|\mathcal{P}| \cdot \max _{\vec{x} \in \mathcal{P}}\left|A \pm A_{\vec{x}}\right| .
\end{aligned}
$$

Note that the assumption $\mathrm{E}_{k}(A) \geqslant k^{2} \mathrm{E}_{k-1}(A)$ implies

$$
\mathrm{E}_{k}(A)=\sum_{\vec{x}}\left|A_{\vec{x}}\right|^{2} \leqslant \sum_{\vec{x}}^{\prime}\left|A_{\vec{x}}\right|^{2}+\left(\begin{array}{c}
k-1 \\
2
\end{array}\right) \mathrm{E}_{k-1}(A) \leqslant 2 \sum_{\vec{x}}^{\prime}\left|A_{\vec{x}}\right|^{2},
$$

where the sum $\sum^{\prime}$ above is taken over $\vec{x}=\left(x_{1}, \ldots, x_{k-1}\right)$ with distinct $x_{j}$. Now take $\mathcal{P}$ such that $\Delta<\left|A_{\vec{x}}\right| \leqslant 2 \Delta$ for $\vec{x}=\left(x_{1}, \ldots, x_{k-1}\right) \in \mathcal{P}$, where all $x_{j}, j \in[k]$ are distinct and

$$
\sum_{\vec{x} \in \mathcal{P}}\left|A_{\vec{x}}\right|^{2} \gg \frac{\mathrm{E}_{k}(A)}{\log K}
$$

Of course, such $\mathcal{P}$ exists by the pigeonhole principle and bound (84). Using the last inequality, and recalling (83), we obtain

$$
\frac{|A|^{2} \mathrm{E}_{k}(A)}{\log K} \ll|A|^{2}|\mathcal{P}| \Delta^{2} \ll \mathrm{E}_{k+1}(A) K^{\varepsilon}|A| .
$$


In other words, $\mathrm{E}_{k+1}(A) \gg_{K^{\varepsilon}}|A| \mathrm{E}_{k}(A)$. Put $M=\max \left\{K^{\varepsilon}, k\right\}$. Applying Proposition 20 as well as Theorem 21, we see that $A \approx_{M} \Lambda \dot{+} H$. After that put $A^{\prime}=A \cap(H+\Lambda)$. Then $\left|A^{\prime}\right| \gg_{M}|A|$ and $|H| \gg_{M}|A| / K$. Further, as in the proof of Theorem 22, we get

$$
\begin{gathered}
\sum_{\vec{x},\|x\|=k-1} \mathcal{C}_{k}\left(A^{\prime}\right)(\vec{x}) \mathcal{C}_{k}(H)(\vec{x})=\sum_{s}\left(A^{\prime} \circ H\right)^{k}(s) \geqslant \frac{|H|^{k}\left|A^{\prime}\right|^{k}}{\left|A^{\prime}-H\right|^{k-1}} \gg_{M} \frac{|H|^{k}|A|^{k}}{|\Lambda+H-H|^{k-1}} \\
\gg_{M} \frac{|H|^{k}|A|^{k}}{(|\Lambda||H-H|)^{k-1}} \gg_{M}|A||H|^{k}
\end{gathered}
$$

and hence

$$
\sum_{\vec{x},\|x\|=k-1}\left|A_{\vec{x}}^{\prime}\right|\left|H_{\vec{x}}\right| \gg_{M}|A||H|^{k}
$$

In particular, there are at least $\gg_{M}|H|^{k-1}$ elements $\vec{x} \in H^{k-1}-\Delta(H)$ such that $\left|A_{\vec{x}}^{\prime}\right| \gg_{M}$ $|A|$. We can suppose that the summation in (85) is taken over $\vec{x}=\left(x_{1}, \ldots, x_{k-1}\right)$ with distinct $x_{j}$ because the rest is bounded by

$$
\left(\begin{array}{c}
k-1 \\
2
\end{array}\right) \sum_{x}(A \circ H)^{k-1}(x) \leqslant\left(\begin{array}{c}
k-1 \\
2
\end{array}\right)|H|^{k-1}|A| \ll_{M}|A||H|^{k} .
$$

The last estimate follows from the assumption $K \leqslant|A|^{1-c}$. Choosing any such $\vec{x}$ and using the Cauchy-Schwarz inequality, we obtain

$$
\left|A \pm A_{\vec{x}}\right| \geqslant\left|A^{\prime} \pm A_{\vec{x}}^{\prime}\right| \geqslant \frac{\left|A^{\prime}\right|^{2}\left|A_{\vec{x}}^{\prime}\right|^{2}}{\mathrm{E}\left(A^{\prime}, A_{\vec{x}}^{\prime}\right)} \gg_{M} \frac{|A|^{4}}{\mathrm{E}(A)}=K_{1}|A|
$$

and in view of Katz-Koester trick (29), we see that $\left|D_{\vec{x}}\right|,\left|S_{\vec{x}}\right|$ are huge for large $K_{1}$. This concludes the proof.

Using Proposition 27 one can derive an interesting dichotomy result.

Theorem 28. Let $A \subseteq \mathbf{G}$ be a set, $D=A-A, S=A+A, k \geqslant 2$, and $M \geqslant 1, \varepsilon \in(0,1)$ be real numbers. Put $K=|A|^{k+1} \mathrm{E}_{k}^{-1}(A)$. Suppose that for any vector $\vec{x}=\left(x_{1}, \ldots, x_{k-1}\right)$ with distinct $x_{j}, j \in[k]$ the following holds

$$
\left|D_{\vec{x}}\right| \leqslant M|A| \quad \text { or, similarly, } \quad\left|S_{\vec{x}}\right| \leqslant M|A| \text {. }
$$

Then either $\mathrm{E}_{k}(A) \ll_{M,|A|^{\varepsilon}, k}|A|^{k}$ or $\mathrm{E}(A) \gg_{M,|A|^{\varepsilon}, k}|A|^{3}$. Again, the degree of the polynomial dependence is a function on $\varepsilon$.

Proof. Put $K_{1}=|A|^{3} \mathrm{E}^{-1}(A)$. Suppose, in contrary, that $\mathrm{E}_{k}(A) \gg_{M,|A|^{\varepsilon}, k}|A|^{k}$ and $\mathrm{E}(A) \ll_{M,|A|^{\varepsilon}, k}|A|^{3}$. Then $K \ll_{M,|A|^{\varepsilon}, k}|A|$ and $K_{1} \gg_{M,|A|^{\varepsilon}, k} 1$. Trivially, $\mathrm{E}_{k}(A) \leqslant$ $|A|^{k-2} \mathrm{E}(A)$ and hence

$$
|A| \gg_{M,|A|^{\varepsilon}, k} K \geqslant K_{1} \gg_{M,|A|^{\varepsilon}, k} 1 \text {. }
$$


Finally, by the upper bound for the parameter $K$ we see that the number $c$ which is defined as $K=|A|^{1-c}$ can by taken depending on $\varepsilon$ only. Thus, everything follows from Proposition 27, the only thing we need to consider is the situation when $\mathrm{E}_{k}(A) \leqslant$ $k^{2} \mathrm{E}_{k-1}(A)$. But in the case

$$
|A|^{k} \leqslant \mathrm{E}_{k}(A) \leqslant k^{2} \mathrm{E}_{k-1}(A) \leqslant k^{2}|A|^{k-3} \mathrm{E}(A) .
$$

In other words $\mathrm{E}(A) \gg_{k}|A|^{3}$ and this concludes the proof.

Thus, if $\left|D_{\vec{x}}\right|,\left|S_{\vec{x}}\right|$ are not much larger than $|A|$ then either $A$ is close to what we called a "random sumset" or, on the contrary, is very structured. Clearly, the both situations are realized.

\section{Energies of sumsets}

Let $A \subseteq \mathbf{G}$ be a set. Throughout the section we put $D=A-A$ and $S=A+A$. As was explained in the introduction one can hope to prove a good lower bound for $\mathrm{E}_{3}(D), \mathrm{E}_{3}(S)$. It will be done in Theorem 30 below but before this we formulate a simple preliminary lower bound for $\mathrm{E}_{k}^{D}(D), \mathrm{E}_{k}^{D}(S)$. Similar lower bounds for $\mathrm{E}_{2}^{D}(D), \mathrm{E}_{2}^{D}(S)$ were given in Corollary 5.6 of paper [21]. Further, it was proved in [28] (see Remark 8) that $\sigma_{k+1}(D) \geqslant\left|A^{k}-\Delta(A)\right|$. Now we obtain a similar lower bound for $\mathrm{E}_{k}^{D}(D)$. Recall that by $\mathrm{E}_{1}^{D}(D)$ we mean $\sigma_{3}(D)=\sigma_{D}(D)$, that is $\sum_{x \in D}(D \circ D)(x)$.

Proposition 29. Let $A \subseteq \mathbf{G}$ be a set. Put $D=A-A, S=A+A$. Then for all $k \geqslant 1$ one has

$$
\mathrm{E}_{k}^{D}(D) \geqslant\left|A^{k+1}-\Delta(A)\right| \geqslant|A-A||A|^{k},
$$

and, similarly,

$$
\begin{gathered}
\sum_{x} S(x)(S * D)^{k}(x) \geqslant\left|A^{k+1}+\Delta(A)\right| \geqslant|A|^{k} \max \{|A-A|,|A+A|\}, \\
\mathrm{E}_{k}^{D}(S) \geqslant|A|^{k-1}\left|A^{2}+\Delta(A)\right| \geqslant|A|^{k} \max \{|A-A|,|A+A|\} .
\end{gathered}
$$

Proof. The second estimates in (86), (87) follow from Lemma 10. Further, it is easy to get (or see e.g. [28]) that

$$
\begin{gathered}
\left|A^{k+1}-\Delta(A)\right| \leqslant \sum_{x_{1}, \ldots, x_{k+1}} D\left(x_{1}\right) \ldots D\left(x_{k+1}\right) \prod_{i \neq j} D\left(x_{i}-x_{j}\right) \leqslant \\
\leqslant \sum_{x_{1}, \ldots, x_{k+1}} D\left(x_{1}\right) \ldots D\left(x_{k+1}\right) D\left(x_{1}-x_{2}\right) \ldots D\left(x_{1}-x_{k+1}\right)=\sum_{x} D(x)(D \circ D)^{k}(x)=\mathrm{E}_{k}^{D}(D)
\end{gathered}
$$


as required. Similarly,

$$
\begin{gathered}
\left|A^{k+1}+\Delta(A)\right| \leqslant \sum_{x_{1}, \ldots, x_{k+1}} S\left(x_{1}\right) \ldots S\left(x_{k+1}\right) \prod_{i \neq j} D\left(x_{i}-x_{j}\right) \leqslant \\
\leqslant \sum_{x_{1}, \ldots, x_{k+1}} S\left(x_{1}\right) \ldots S\left(x_{k+1}\right) D\left(x_{1}-x_{2}\right) \ldots D\left(x_{1}-x_{k+1}\right)=\sum_{x} S(x)(S * D)^{k}(x) .
\end{gathered}
$$

Finally, by Lemma 10, we get

$$
\begin{aligned}
\mathrm{E}_{k}^{D}(S) & =\sum_{s \in D}(S \circ S)^{k}(s) \geqslant \sum_{s \in D}\left|A+A_{s}\right|^{k} \geqslant|A|^{k-1} \sum_{s \in D}\left|A+A_{s}\right|= \\
& =|A|^{k-1}\left|A^{2}+\Delta(A)\right| \geqslant|A|^{k} \max \{|A-A|,|A+A|\} .
\end{aligned}
$$

This completes the proof.

Interestingly, that some sort of sumset, namely, $A^{n} \pm \Delta(A)$ gives a lower bound for an energy, although, usually, an energy provides lower bounds for cardinality of sumsets via the Cauchy-Schwarz inequality. The trick allows to obtain a series of results in [28] - [30]. Although bounds (86), (87) are very simple they can be tight in some cases. For example, take $A$ to be a dissociated set or, in contrary, a very structural set as a subspace.

Now we formulate the main result of the section concerning lower bounds for some energies of sumsets/difference sets. Again we need in lower bounds on $\mathrm{E}_{k}(A)$ in Theorem 30 below to be separated from the "random sumset" case.

Theorem 30. Let $A \subseteq \mathbf{G}$ be a set. Take two sets $D, S$ such that $D=A-A, S=A+A$. Then

$$
\mathrm{E}_{3}^{2}(D, A, A), \quad \mathrm{E}_{3}^{2}(S, A, A) \geqslant \frac{|A|^{13}}{|A-A|^{2} \mathrm{E}(A)},
$$

and

$$
\left(\mathrm{E}_{3}^{D}(D)\right)^{4} \geqslant \max \left\{|D|^{12}, \frac{|A|^{45}}{\mathrm{E}^{9}(A)|D|^{2}}\right\}, \quad\left(\mathrm{E}_{3}^{D}(S)\right)^{4} \geqslant \max \left\{|S|^{12}, \frac{|A|^{45}}{\mathrm{E}^{9}(A)|D|^{2}}\right\} .
$$

Further, let $\beta, \gamma \in[0,1]$ be real numbers, $\beta \leqslant 1 / 2$. If $A$ is $(2, \beta, \gamma)$-connected then

$$
\mathrm{E}_{3}^{2}(D, A, A), \quad \mathrm{E}_{3}^{2}(S, A, A) \gg \gamma|A|^{5} \mathrm{E}(A) .
$$

Suppose that $A$ is $(3 / 2, \beta, \gamma)$ and $(2, \beta, \gamma)$-connected, correspondingly. Then

$$
\mathrm{E}_{3}^{D}(D), \mathrm{E}_{3}^{D}(S) \gg \gamma \frac{|A|^{33 / 4} \mathrm{E}_{3 / 2}(A)}{\mathrm{E}^{9 / 4}(A) \log |A|}, \quad \mathrm{E}_{3}^{D}(D), \mathrm{E}_{3}^{D}(S) \gg \gamma \frac{|A|^{17 / 2}}{\mathrm{E}^{3 / 2}(A) \log |A|},
$$

correspondingly. 
Proof. Write $\mathrm{E}=\mathrm{E}(A), \mathrm{E}_{3}=\mathrm{E}_{3}(A)$, and $a=|A|$. Let us obtain bounds (88), (90). Using Lemma 13 , we find $A^{\prime},\left|A^{\prime}\right| \geqslant|A| / 2$ such that estimate (33) takes place. As in the proof of inequalities (68), (71), we get

$$
a^{2}\left|A_{s}^{\prime}\right|^{2} \leqslant \mathrm{E}\left(A, A_{s}^{\prime}\right)\left|A \pm A_{s}^{\prime}\right| \leqslant \frac{2 \mathrm{E}}{a}\left|A_{s}^{\prime}\right|\left|A \pm A_{s}\right| .
$$

Multiplying the last inequality by $\left|A_{s}^{\prime}\right|$, summing over $s$ and using Katz-Koester trick, we have

$$
a^{2} \mathrm{E}_{3}\left(A^{\prime}\right) \ll a^{-1} \mathrm{E} \cdot \mathrm{E}_{3}(D, A)
$$

and similar for $S$. On the other hand by the second part of Lemma 8, we obtain

$$
\left(\frac{a^{4}}{|A-A|}\right)^{2} a^{2} \ll \mathrm{E}^{2}\left(A^{\prime}\right) a^{2} \ll \mathrm{E}_{3}\left(A^{\prime}\right) \cdot \mathrm{E}_{3}(D, A)
$$

and using the first part of the lemma, we have the same bound for the set $S$

$$
\left(\frac{a^{2}}{|A-A|}\right)^{2} a^{6} \ll \sigma_{\tilde{D}}^{2}\left(A^{\prime}\right)\left(a^{\prime}\right)^{2}\left(\frac{\left(a^{\prime}\right)^{2}}{2|D|}\right)^{2} \leqslant \mathrm{E}_{3}\left(A^{\prime}\right) \sum_{x \in \tilde{D}}\left|S_{x}\right|\left|A_{x}\right|^{2} \leqslant \mathrm{E}_{3}\left(A^{\prime}\right) \cdot \mathrm{E}_{3}(S, A),
$$

where $\tilde{D}:=\left\{x \in D:\left|A_{x}^{\prime}\right| \geqslant\left(a^{\prime}\right)^{2} / 2|D|\right\}, \sigma_{\tilde{D}}\left(A^{\prime}\right) \geqslant\left(a^{\prime}\right)^{2} / 2$ (for details, see [30]). Another way to prove the same is to use Lemma 14 with $A=B=A^{\prime}, \psi(x)=\left(A^{\prime} \circ A^{\prime}\right)(x)$. Combining (93) and (94), (95), we get

$$
\mathrm{E}_{3}^{2}(D, A), \mathrm{E}_{3}^{2}(S, A) \gg \frac{|A|^{13}}{|A-A|^{2} \mathrm{E}(A)}
$$

Using the tensor trick (see [37] or [33]), we have (88). If $A$ is $(2, \beta, \gamma)$-connected then

$$
\mathrm{E}\left(A^{\prime}\right) \gg \gamma \mathrm{E}(A)
$$

and combining the last inequality with (93) and the second bound from (94), we get (90) (to obtain lower bound for $\mathrm{E}_{3}^{2}(S, A)$ one should use Lemma 14).

It remains to prove (89) and (91). Returning to (92)-(93), we obtain

$$
a^{3} \mathrm{E}_{3}\left(A^{\prime}\right) \ll \mathrm{E} \sum_{s}\left|A_{s}^{\prime}\right|^{2}\left|A^{\prime} \pm A_{s}^{\prime}\right|
$$

or, by the Hölder inequality

$$
a^{9} \mathrm{E}_{3}\left(A^{\prime}\right) \ll \mathrm{E}^{3} \mathrm{E}_{3}^{D}(D) .
$$

On the other hand, by Lemma 8 for any $P \subseteq A^{\prime}-A^{\prime}$, we have

$$
a^{2} \sigma_{P}^{2}\left(A^{\prime}\right) \ll \mathrm{E}_{3}\left(A^{\prime}\right) \sum_{s \in P}\left|A^{\prime} \pm A_{s}^{\prime}\right|
$$


Applying the Hölder inequality, we get

$$
a^{6} \sigma_{P}^{6}\left(A^{\prime}\right) \ll \mathrm{E}_{3}^{3}\left(A^{\prime}\right)|P|^{2} \mathrm{E}_{3}^{D}(D)
$$

and similarly for $S$. Now choose $P \subseteq A^{\prime}-A^{\prime}$ such that $P=\left\{s \in A^{\prime}-A^{\prime}: \rho<\left|A_{s}^{\prime}\right| \leqslant 2 \rho\right\}$ for some positive number $\rho$ and such that

$$
\sum_{s \in P}\left|A_{s}^{\prime}\right|^{3 / 2} \gg \frac{\mathrm{E}_{3 / 2}\left(A^{\prime}\right)}{\log a} .
$$

Of course, the set $P$ exists by Dirichlet principle. Combining the last inequality with (97), we obtain

$$
a^{6} \mathrm{E}_{3 / 2}^{4}\left(A^{\prime}\right) \log ^{-4} a \ll a^{6}\left(|P| \rho^{3 / 2}\right)^{4} \ll \mathrm{E}_{3}^{3}\left(A^{\prime}\right) \mathrm{E}_{3}^{D}(D) .
$$

Using estimates (96), (98), we have

$$
\left(\mathrm{E}_{3}^{D}(D)\right)^{4} \mathrm{E}^{9} \gg a^{33} \mathrm{E}_{3 / 2}^{4}\left(A^{\prime}\right) \log ^{-4} a .
$$

Thus

$$
\left(\mathrm{E}_{3}^{D}(D)\right)^{4},\left(\mathrm{E}_{3}^{D}(S)\right)^{4} \gg \frac{a^{45}}{\mathrm{E}^{9}|D|^{2} \log ^{4} a} .
$$

Applying the tensor trick again, we get (89). To obtain (91) recall that $A$ is $(3 / 2, \beta, \gamma)-$ connected set. Hence by (99), we obtain

$$
\left(\mathrm{E}_{3}^{D}(D)\right)^{4} \mathrm{E}^{9} \gg \gamma^{4} a^{33} \mathrm{E}_{3 / 2}^{4}(A) \log ^{-4} a
$$

and the first formula of (91) follows. Further, because $A$ is $(2, \beta, \gamma)$-connected set then using Lemma 12 for $A^{\prime}$ as well as (99), we have

$$
\left(\mathrm{E}_{3}^{D}(D)\right)^{2} \mathbf{E}^{3} \gg \gamma^{2} a^{17} \log ^{-2} a
$$

and the last estimate coincide with the second inequality in (91). This completes the proof.

From (89), the definition of the number $K$ as $|D|=K|A|$ and the assumption $\mathrm{E}(A) \ll$ $|A|^{3} / K$, we get

$$
\mathrm{E}_{3}(D) \gg K^{7 / 4}|A|^{4}
$$

An upper bound here is $K^{2}|A|^{4}$ and it follows from the main example of section 4 , that is $\mathbf{G}=\mathbb{F}_{2}^{n}, A=H \dot{+} \Lambda$. Note also that the second inequality in formula (91) is weak but do not depends on the size of $A-A$ or on energy $\mathrm{E}_{3 / 2}(A)$.

As for dual quantities $\mathrm{T}_{k}(D), \mathrm{T}_{k}(S)$, our example $A=H \dot{+} \Lambda$ shows that there are not nontrivial lower bounds for $\mathrm{T}_{k}(A \pm A)$, in general, which are better than simple consequences of Katz-Koester

$$
\mathrm{T}_{k}(D) \geqslant|A|^{2} \mathrm{~T}_{k-1}(D) \geqslant \ldots \geqslant|A|^{2(k-2)} \mathrm{E}(D) \geqslant|A|^{2 k-2}|D|,
$$




$$
\mathrm{T}_{k}(S) \geqslant|A|^{2} \mathrm{~T}_{k-1}(S, \ldots, S, D) \geqslant \ldots \geqslant|A|^{2(k-2)} \mathrm{E}(D) \geqslant|A|^{2 k-2}|D|
$$

(just use $(D \circ D)(x) \geqslant|A| D(x)$ and $(S \circ S)(x) \geqslant|A| D(x))$. The reason is that the structure of $A \pm A$ is similar to the structure of $A$ in the case, of course. Nevertheless, it was proved in [28], Lemma 3 that

$$
|A|^{4 k} \leqslant \mathrm{E}_{2 k}(A) \mathrm{T}_{k}(A \pm A),
$$

and also in [33], Note 6.6 that

$$
\left(\frac{\sum_{x \in P}(A \circ A)(x)}{|A|}\right)^{4 k} \leqslant \mathrm{E}_{2 k}(A) \mathrm{T}_{k}(P),
$$

where $P \subseteq D$ is any set. So, if we know something on $\mathrm{E}_{2 k}(A)$ then it gives us a new information about $\mathrm{T}_{k}(A \pm A)$. Trivially, formula (101) implies that $\mathrm{T}_{k}(A \pm A) \geqslant|A|^{2 k+2} / \mathrm{E}(A)$. Again, the last inequality is sharp as our main example $A=H \dot{+} \Lambda$ shows.

Vsevolod F. Lev asked the author about an analog of (102) for different sets $A$ and $B$. Proposition 31 below is our result in the direction. The proof is in spirit of [33]. For simplicity we consider the case $k=2$ only. The case of greater powers of two is considered similarly if one take $M^{2}, M^{4}, \ldots$ or just see the proof of Theorem 6.3 from [33] (the case of any $k$ ). We do not insert the full proof because we avoid to use the operators from [32], [33] in the paper which is considered to be elementary.

Proposition 31. Let $k \geqslant 2$ be a power of two, $A, B \subseteq \mathbf{G}$ be two sets, and $P \subseteq A-B$. Then

$$
\left(\frac{\sum_{x \in P}(A \circ B)(x)}{|A|^{1 / 2}|B|^{1 / 2}}\right)^{4 k} \leqslant \mathrm{E}_{2 k}(A, \ldots, A, B, \ldots, B) \mathrm{T}_{k}(P) .
$$

Proof. Let $k=2$. Define the matrix

$$
M(x, y)=P(x-y) A(x) B(y)
$$

and calculate its rectangular norm (e.g., see section 7)

$$
\begin{gathered}
\lambda_{1}^{4}(M) \leqslant \sum_{j} \lambda_{j}^{4}(M)=\sum_{x, y, x^{\prime}, y^{\prime}} M(x, y) M\left(x^{\prime}, y\right) M\left(x, y^{\prime}\right) M\left(x^{\prime}, y^{\prime}\right)= \\
=\sum_{x, x^{\prime} \in A} \sum_{y, y^{\prime} \in B} P(x-y) P\left(x^{\prime}-y\right) P\left(x-y^{\prime}\right) P\left(x^{\prime}-y^{\prime}\right)= \\
=\sum_{\alpha, \beta, \gamma} \mathcal{C}_{4}(B, A, A, B)(\alpha, \beta, \gamma) P(\alpha) P(\beta) P(\alpha-\gamma) P(\beta-\gamma),
\end{gathered}
$$

where $\lambda_{j}(M)$ the singular numbers of $M$. Clearly,

$$
\lambda_{1}(M) \geqslant \frac{\sum_{x \in P}(A \circ B)(x)}{|A|^{1 / 2}|B|^{1 / 2}} .
$$


Thus, by the Cauchy-Schwarz inequality, we get

$$
\left(\frac{\sum_{x \in P}(A \circ B)(x)}{|A|^{1 / 2}|B|^{1 / 2}}\right)^{8} \leqslant \mathrm{E}_{4}(A, A, B, B) \mathrm{E}(P)
$$

as required. This completes the proof.

We end this section showing that there is a different way to prove our Theorem $30 \mathrm{using}$ slightly bigger sets $D_{x}$ or $S_{x}$ not $A \pm A_{x}$. The proof based on a lemma, which demonstrates, in particular, that $A \pm A$ contains approximately $|A|^{3} \mathrm{E}^{-1}(A)$ almost disjoint translates of $A$, roughly. In the proof we use arguments from [1].

Lemma 32. Let $A, B \subseteq \mathbf{G}$ be two sets. Then there are

$$
s \geqslant 2^{-4}|A||B|^{2} \mathrm{E}^{-1}(A, B)
$$

disjoint sets $A_{j} \subseteq A+b_{j},\left|A_{j}\right| \geqslant|A| / 2, b_{j} \in B, j \in[s]$.

Moreover, for any set $S \subseteq A+B$ put $\sigma=\sum_{x \in S}(A * B)(x)$. Suppose that $\sigma \geqslant 16|B|$. Then there are

$$
s \geqslant 2^{-8} \sigma^{3}|A|^{-2}|B|^{-1} \mathrm{E}^{-1}(A, B)
$$

disjoint sets $S_{j} \subseteq S \cap\left(A+b_{j}\right),\left|S_{j}\right| \geqslant 2^{-3} \sigma|B|^{-1}, b_{j} \in B, j \in[s]$.

Proof. Let us begin with (103). Put $S=A+B$. Our arguments is a sort of an algorithm. At the first step of the algorithm take $A_{1}=A+b$, where $b \in B$ is any element of $B$. Suppose that we have constructed $k$ disjoint sets $A_{1}, \ldots, A_{k}$. If there is $b \in B$ such that $\left|(A+b) \backslash \bigsqcup_{j=1}^{k} A_{j}\right| \geqslant|A| / 2$ then put $A_{k+1}=(A+b) \backslash \bigsqcup_{j=1}^{k} A_{j}$ and take $b_{k+1}=b$. Suppose that our algorithm stops after $s$ steps. If $s \geqslant|B| / 2$ then we are done. Put $U=\bigsqcup_{j=1}^{s} A_{j}$ and $B_{*}=B \backslash\left\{b_{1}, \ldots, b_{s}\right\}$. Then $s|A| / 2<|U| \leqslant s|A|$ and $\left|B_{*}\right| \geqslant|B| / 2$. We have

$$
2^{-2}|A||B| \leqslant 2^{-1}|A|\left|B_{*}\right| \leqslant \sum_{x}(A \circ U)(x) B_{*}(x) .
$$

Using the Cauchy-Schwarz inequality, we obtain

$$
2^{-4}|A|^{2}|B|^{2} \leqslant \mathrm{E}(A, B)|U| \leqslant \mathrm{E}(A, B) s|A|
$$

and the required lower bound for $s$ follows.

Let us prove the second part of the lemma. Put $a=|A|, b=|B|$. First of all note that

$$
\sigma=\sum_{x \in S}(A * B)(x)=\sum_{x \in B}|S \cap(A+x)|
$$

and hence there is $x \in B$ such that $|S \cap(A+x)| \geqslant \sigma b^{-1}$. Put $b_{1}=x$, and let $S_{1} \subseteq$ $S \cap\left(A+b_{1}\right)$ be an arbitrary set of size $\left\lceil\varepsilon \sigma b^{-1}\right\rceil$, where $\varepsilon=1 / 8$. After that using the arguments as above, we construct a family of disjoint sets $S_{j} \subseteq S \cap\left(A+b_{j}\right),\left|S_{j}\right| \geqslant \varepsilon \sigma b^{-1}$, 
$b_{j} \in B, j \in[s]$. If $s \geqslant \sigma /(4 a)$ then we are done. If not then put $U=\bigsqcup_{j=1}^{s} S_{j}$ and $B_{*}=B \backslash\left\{b_{1}, \ldots, b_{s}\right\}$. We have $B_{*} \subseteq B_{*}^{\prime} \bigcup B_{*}^{\prime \prime}$, where

$$
\begin{gathered}
B_{*}^{\prime}=\left\{x \in B_{*}:(A \circ U)(x) \geqslant \varepsilon \sigma b^{-1}\right\}, \\
B_{*}^{\prime \prime}=\left\{x \in B_{*}:|(A+x) \backslash S| \geqslant a-2 \varepsilon \sigma b^{-1}\right\} .
\end{gathered}
$$

Further, because

$$
\left(a-2 \varepsilon \sigma b^{-1}\right)\left|B_{*}^{\prime \prime}\right| \leqslant \sum_{x \in B_{*}^{\prime \prime}}|(A+b) \backslash S| \leqslant \sum_{x \in B}|(A+x) \backslash S| \leqslant a b-\sum_{x \in B}|(A+x) \bigcap S|=a b-\sigma
$$

we see that $\left|B_{*}^{\prime \prime}\right| \leqslant(b-\sigma / a)(1+4 \varepsilon \sigma /(a b))$. Thus,

$$
\left|B_{*}^{\prime}\right| \geqslant b-s-(b-\sigma / a)(1+4 \varepsilon \sigma /(a b)) \geqslant \sigma / a-4 \varepsilon \sigma / a-s \geqslant \sigma / 4 a .
$$

Finally, we obtain

$$
\varepsilon \sigma b^{-1} \cdot \sigma / 4 a \leqslant \sum_{x}(A \circ U)(x) B_{*}^{\prime}(x) \leqslant \sum_{x}(A \circ U)(x) B(x)
$$

and hence, in view of the condition $\sigma \geqslant 16 b$ the following holds

$$
\left(\varepsilon \sigma b^{-1}\right)^{2} \cdot(\sigma / 4 a)^{2} \leqslant|U| \mathrm{E}(A, B) \leqslant\left(2 \varepsilon \sigma b^{-1}\right) s \mathrm{E}(A, B) .
$$

Whence, $s \geqslant 2^{-8} \sigma^{3} a^{-2} b^{-1} \mathrm{E}^{-1}(A, B)$. This concludes the proof.

Now let us show how to get (90), for example. Applying Lemma 32 with $A=A$, $B=-A$, we obtain

$$
\mathrm{E}_{3}(D, A, A) \geqslant \sum_{j=1}^{s} \mathrm{E}_{3}\left(A_{j}, A, A\right) \gg|A|^{3} \mathrm{E}^{-1}(A) \mathrm{E}_{3}(A)
$$

provided by $(3, \beta, \gamma)$-connectedness assumptions, $\beta, \gamma \gg 1$ (by the way bound (105) is tight as our main example $H \dot{+} \Lambda$ shows). On the other hand, we have by Lemma 8 that

$$
\mathrm{E}^{2}(A)|A|^{2} \leqslant \mathrm{E}_{3}(A) \mathrm{E}_{3}(D, A, A)
$$

Combining the last two bounds, we get (90).

Using similar arguments and the second part of Lemma 32, we obtain the following consequence, which shows, in particular, that the popular difference sets [15], [37] have some structure in the sense that they have large energy of some sort.

Corollary 33. Let $A \subseteq \mathbf{G}$ be a set, $P \subseteq A-A$. Suppose that $A$ is a $(3, \beta, \gamma)$-connected set, $\beta \leqslant 2^{-3} \sigma_{P}(A)|A|^{-1}$. Then

$$
\mathrm{E}_{3}(P, A, A) \geqslant 2^{-9} \gamma^{1 / 2} \sigma_{P}^{5}(A) \mathrm{E}(A)|A|^{-9} .
$$


Proof. Let $\sigma=\sigma_{P}(A)$. On the one hand, using Lemma 32 with $A=A, B=-A$, we construct the family of disjoint sets $P_{j} \subseteq P \cap\left(A-a_{j}\right), a_{j} \in A,\left|P_{j}\right| \geqslant 2^{-3} \sigma|A|^{-1}$, the number $s$ satisfies (104). Put $A_{j}=P_{j}+a_{j} \subseteq A$. Thus, by connectedness of our set $A$, we have

$$
\mathrm{E}_{3}(P, A) \geqslant \sum_{j=1}^{s} \mathrm{E}_{3}\left(P_{j}, A\right)=\sum_{j=1}^{s} \mathrm{E}_{3}\left(A_{j}, A\right) \geqslant \gamma\left(\frac{\left|A_{j}\right|}{|A|}\right)^{6} \mathrm{E}_{3}(A) \geqslant 2^{-18} \gamma \frac{\sigma^{6}}{|A|^{12}} \mathrm{E}_{3}(A) .
$$

On the other hand, applying Lemma 8, we get

$$
\sigma^{4} \mathrm{E}^{2}(A)|A|^{-6} \leqslant \mathrm{E}_{3}(A) \mathrm{E}_{3}(P, A) .
$$

Combining the last two inequalities, we obtain bound (106). This concludes the proof.

\section{On Gowers norms}

The notion of Gowers norms was introduced in papers [14, 15]. At the moment it is a very important tool of investigation in wide class of problems of additive combinatorics (see e.g. [8] - [15], [20], [24]) as well as in ergodic theory (see e.g. [2], [6], [16], [17], [35], [36], [38], [39], [40]). Recall the definitions.

Let $G$ be a finite set, and $N=|G|$. Let also $d$ be a positive integer, and

$$
\{0,1\}^{d}=\left\{\omega=\left(\omega_{1}, \ldots, \omega_{d}\right): \omega_{j} \in\{0,1\}, j=1,2, \ldots, d\right\}
$$

be the ordinary $d$-dimensional cube. For $\omega \in\{0,1\}^{d}$ denote by $|\omega|$ the sum $\omega_{1}+\cdots+\omega_{d}$. Let also $\mathcal{C}$ be the operator of complex conjugation. Let $\vec{x}=\left(x_{1}, \ldots, x_{d}\right), \vec{x}^{\prime}=\left(x_{1}^{\prime}, \ldots, x_{d}^{\prime}\right)$ be two arbitrary vectors from $G^{d}$. By $\vec{x}^{\omega}=\left(\vec{x}_{1}^{\omega}, \ldots, \vec{x}_{d}^{\omega}\right)$ denote the vector

$$
\vec{x}_{i}^{\omega}=\left\{\begin{array}{cc}
x_{i} & \text { if } \omega_{i}=0 \\
x_{i}^{\prime} & \text { if } \omega_{i}=1
\end{array}\right.
$$

Thus $\vec{x}^{\omega}$ depends on $\vec{x}$ and $\vec{x}^{\prime}$.

Let $f: G^{d} \rightarrow \mathbb{C}$ be an arbitrary function. We will write $f(\vec{x})$ for $f\left(x_{1}, \ldots, x_{d}\right)$.

Definition 34. Gowers $U^{d}$-norm (or $d$-uniformity norm) of the function $f$ is the following expression

$$
\|f\|_{U^{d}}=\left(N^{-2 d} \sum_{\vec{x} \in G^{d}} \sum_{\vec{x}^{\prime} \in G^{d}} \prod_{\omega \in\{0,1\}^{d}} \mathcal{C}^{|\omega|} f\left(\vec{x}^{\omega}\right)\right)^{1 / 2^{d}} .
$$

A sequence of $2^{d}$ points $\vec{x}^{\omega} \in G^{d}, \omega \in\{0,1\}^{d}$ is called $d$-dimensional cube in $G^{d}$ or just a $d$-dimensional cube. Thus the summation in formula (107) is taken over all cubes of $G^{d}$. For example, $\left\{(x, y),\left(x^{\prime}, y\right),\left(x, y^{\prime}\right),\left(x^{\prime}, y^{\prime}\right)\right\}$, where $x, x^{\prime}, y, y^{\prime} \in G$ is a two-dimensional cube in $G \times G$. In the case Gowers norm is called rectangular norm. 
For $d=1$ the expression above gives a semi-norm but for $d \geqslant 2$ Gowers norm is a norm. In particular, the triangle inequality holds [15]

$$
\|f+g\|_{U^{d}} \leqslant\|f\|_{U^{d}}+\|g\|_{U^{d}}
$$

One can prove also (see [15]) the following monotonicity relation. Let $f_{x_{d}}\left(x_{1}, \ldots, x_{d-1}\right):=$ $f\left(x_{1}, \ldots, x_{d}\right)$. Then

$$
N^{-1} \sum_{x_{d} \in G}\left\|f_{x_{d}}\right\|_{U^{d-1}}^{2^{d-1}} \leqslant\|f\|_{U^{d}}^{2^{d-1}}
$$

for all $d \geqslant 2$.

If $\mathbf{G}=(\mathbf{G},+)$ is a finite Abelian group with additive group operation,$+ N=|\mathbf{G}|$ then one can "project" the norm above onto the group $\mathbf{G}$ and obtain the ordinary ("onedimensional") Gowers norm. In other words, we put the function $f\left(x_{1}, \ldots, x_{d}\right)$ in formula (107) equals "one-dimensional" function $f\left(x_{1}, \ldots, x_{d}\right):=f\left(\operatorname{pr}\left(x_{1}, \ldots, x_{d}\right)\right)$, where $\operatorname{pr}\left(x_{1}, \ldots, x_{d}\right)=x_{1}+\cdots+x_{d}$. Denoting the obtained norm as $U^{d}$, we have an analog of (109), see [15], [37]

$$
\|f\|_{U^{d-1}} \leqslant\|f\|_{U^{d}}
$$

for all $d \geqslant 2$. It is convenient to write

$$
\begin{aligned}
\|f\|_{\mathcal{U}^{d}} & =N^{-d+1} \sum_{\vec{x} \in \mathbf{G}^{d}} \sum_{\vec{x}^{\prime} \in \mathbf{G}^{d}} \prod_{\omega \in\{0,1\}^{d}} \mathcal{C}^{|\omega|} f\left(\operatorname{pr}\left(\vec{x}^{\omega}\right)\right)= \\
& =\sum_{x} \sum_{h_{1}, \ldots, h_{d}} \prod_{\omega \in\{0,1\}^{d}} \mathcal{C}^{|\omega|} f(x+\omega \cdot \vec{h}) .
\end{aligned}
$$

In the case $f=A$, where $A \subseteq \mathbf{G}$ is a set, we have by formula (112) that

$$
\|A\|_{\mathcal{U}^{d}}=\sum_{s_{1}, \ldots, s_{d}}\left|A_{\pi\left(s_{1}, \ldots, s_{d}\right)}\right|
$$

where $\pi\left(s_{1}, \ldots, s_{d}\right)$ is a vector with $2^{d}$ components, namely,

$$
\pi\left(s_{1}, \ldots, s_{d}\right)=\left(\sum_{j=1}^{d} s_{j} \varepsilon_{j}\right), \quad \varepsilon_{j} \in\{0,1\}^{d} .
$$

Note also

$$
\|A\|_{\mathcal{U}^{d+1}}=\sum_{s_{1}, \ldots, s_{d}}\left|A_{\pi\left(s_{1}, \ldots, s_{d}\right)}\right|^{2} .
$$

Further, $\|A\|_{\mathcal{U}^{1}}=\mathrm{E}_{1}(A)=|A|^{2}$ and $\|A\|_{\mathcal{U}^{2}}=\mathrm{E}(A)$.

In definitions (107), (111) we have used the size of the set G/group G. The results of the paper are local, in the sense that they do not use cardinality of the container group $\mathbf{G}$. Thus it is natural to ask about the possibility to obtain an analog of (110), say, without any $N$ in the definition. That is our simple result in the direction. 
Proposition 35. Let $A \subseteq \mathbf{G}$ be a set. Then for any integer $k \geqslant 2$ one has

$$
\|A\|_{\mathcal{U}^{k+1}} \geqslant \frac{\|A\|_{\mathcal{U}^{k}}^{(3 k-2) /(k-1)}}{\|A\|_{\mathcal{U}^{k-1}}^{2 k /(k-1)}} .
$$

In particular,

$$
\|A\|_{\mathcal{U}^{3}} \geqslant \frac{\mathrm{E}^{4}(A)}{|A|^{8}}
$$

Proof. We have

$$
\begin{gathered}
\|A\|_{\mathcal{U}^{k}}=\sum_{s_{1}, \ldots, s_{k}}\left|A_{\pi\left(s_{1}, \ldots, s_{k}\right)}\right|=\sum_{s_{1}, \ldots, s_{k-1}} \sum_{s_{k}} \sum_{z} A_{\pi\left(s_{1}, \ldots, s_{k-1}\right)}(z) A_{\pi\left(s_{1}, \ldots, s_{k-1}\right)}\left(z+s_{k}\right)= \\
=\sum_{s_{1}, \ldots, s_{k-1}} \sum_{z} A_{\pi\left(s_{1}, \ldots, s_{k-1}\right)}(z) \cdot\left|A_{\pi\left(s_{1}, \ldots, s_{k-1}\right)}\right| .
\end{gathered}
$$

Thus, if the summation in (116) is taken over the set

$$
Q_{k}:=\left\{\left(s_{1}, \ldots, s_{k-1}\right):\left|A_{\pi\left(s_{1}, \ldots, s_{k-1}\right)}\right| \geqslant\|A\|_{\mathcal{U}^{k}}\left(2 k\|A\|_{\mathcal{U}^{k-1}}\right)^{-1}\right\}
$$

then it gives us $(1-1 / 2 k)$ proportion of the norm $\|A\|_{\mathcal{U}^{k}}$. Let us estimate the size of $Q_{k}$. Clearly,

$$
\left|Q_{k}\right|\|A\|_{\mathcal{U}^{k}}\left(2 k\|A\|_{\mathcal{U}^{k-1}}\right)^{-1} \leqslant \sum_{s_{1}, \ldots, s_{k-1}}\left|A_{\pi\left(s_{1}, \ldots, s_{k-1}\right)}\right|=\|A\|_{\mathcal{U}^{k-1}}
$$

and whence $\left|Q_{k}\right| \leqslant 2 k\|A\|_{\mathcal{U}^{k-1}}^{2}\|A\|_{\mathcal{U}^{k}}^{-1}$. Certainly, the same bound holds for cardinality of any set of tuples $\left(s_{i_{1}}, \ldots, s_{i_{k-1}}\right)$ defined similar to (117) and having the size $k-1$. Hence, by the standard projection results, see e.g. [5], we see that the summation in (116) is taken over a set $\mathcal{S}$ of vectors $\left(s_{1}, \ldots, s_{k}\right)$ of size at most $\left(2 k\|A\|_{\mathcal{U}^{k-1}}^{2}\|A\|_{\mathcal{U}^{k}}^{-1}\right)^{k /(k-1)}$. Returning to (116) and using the Cauchy-Schwarz inequality as well as formula (113), we obtain

$$
\begin{aligned}
2^{-2}\|A\|_{\mathcal{U}^{k}}^{2} \leqslant & \left(\sum_{\left(s_{1}, \ldots, s_{k}\right) \in \mathcal{S}}\left|A_{\pi\left(s_{1}, \ldots, s_{k}\right)}\right|\right)^{2} \leqslant|\mathcal{S}| \sum_{s_{1}, \ldots, s_{k}}\left|A_{\pi\left(s_{1}, \ldots, s_{k}\right)}\right| \leqslant \\
& \leqslant\left(2 k\|A\|_{\mathcal{U}^{k-1}}^{2}\|A\|_{\mathcal{U}^{k}}^{-1}\right)^{k /(k-1)}\|A\|_{\mathcal{U}^{k+1}} .
\end{aligned}
$$

The last inequality implies that

$$
\|A\|_{\mathcal{U}^{k+1}} \geqslant C_{k} \frac{\|A\|_{\mathcal{U}^{k}}^{(3 k-2) /(k-1)}}{\|A\|_{\mathcal{U}^{k-1}}^{2 k /(k-1)}},
$$

where $0<C_{k}<1$ depends on $k$ only. Using the tensor trick we obtain the result. This completes the proof. 
Remark 36. Estimate (114) is sharp as an example of a sufficiently dense random subset of a group $\mathbf{G}$ shows. For higher Gowers norms one can obtain by induction a similar sharp inequality $\|A\|_{\mathcal{U}^{k}} \geqslant \mathrm{E}(A)^{2^{k}-k-1}|A|^{-\left(3 \cdot 2^{k}-4 k-4\right)}$. It demonstrates expected exponential (in terms of $\mathrm{E}(A))$ growth of the norms.

In the next section we will need in a statement, which is generalizes lower bound for $U^{3}$-norm (115).

Lemma 37. Let $A, B \subseteq \mathbf{G}$ be two sets. Then

$$
\sum_{s_{1}, s_{2}}\left(\sum_{x} A(x) B\left(x+s_{1}\right) A\left(x+s_{2}\right) B\left(x+s_{1}+s_{2}\right)\right)^{2} \geqslant \frac{\mathrm{E}^{4}(A, B)}{|A|^{4}|B|^{4}} .
$$

Proof. We use the same arguments as in the proof of Proposition 35. One has

$$
\begin{aligned}
\mathrm{E}(A, B) & =\sum_{s_{1}, s_{2}} \sum_{x} A(x) B\left(x+s_{1}\right) A\left(x+s_{2}\right) B\left(x+s_{1}+s_{2}\right)= \\
& =\sum_{x} \sum_{s_{1}} B_{s_{1}}^{A}(x)\left|B_{s_{1}}^{A}\right|=\sum_{x} \sum_{s_{2}} A_{s_{2}}(x)\left|B_{s_{2}}\right| .
\end{aligned}
$$

Because of $\sum_{s}\left|A_{s}\right|=|A|^{2}, \sum_{s}\left|B_{s}\right|=|B|^{2}$, we get for the set $\mathcal{S}$ above that $|\mathcal{S}| \ll\left(|A|^{2}|B|^{2} \mathrm{E}^{-1}(A, B)\right)^{2}$. Thus

$$
\mathrm{E}^{2}(A, B) \ll|\mathcal{S}| \sum_{s_{1}, s_{2}}\left(\sum_{x} A(x) B\left(x+s_{1}\right) A\left(x+s_{2}\right) B\left(x+s_{1}+s_{2}\right)\right)^{2}
$$

and the result follows.

Using (115) and the Cauchy-Schwarz inequality, we have a consequence.

Corollary 38. Let $A \subseteq \mathbf{G}$ be a set and $|A-A| \leqslant K|A|$ or $|A+A| \leqslant K|A|$. Then

$$
\|A\|_{\mathcal{U}^{3}} \geqslant \frac{|A|^{4}}{K^{4}}
$$

Inequality (115) gives us a relation between $\|A\|_{\mathcal{U}^{3}}=\sum_{s} \mathrm{E}\left(A_{s}\right)$ and $\mathrm{E}(A)$. W.T. Gowers (see [15]) constructed a set $A$ having a random behavior in terms of $\mathrm{E}(A)$ (more precisely, he constructed a uniform set, that is having small Fourier coefficients, see [15]) such that for all $s$ the sets $A_{s}$ have non-random (non-uniform) behavior in terms of $\mathrm{E}\left(A_{s}\right)$. Nevertheless, it is natural to ask about the possibility to find an $s \neq 0$ with a weaker notion of randomness, that is $\mathrm{E}\left(A_{s}\right) \ll\left|A_{s}\right|^{3-c}, c>0$. This question was asked to the author by $\mathrm{T}$. Schoen. We give an affirmative answer on it. 
Theorem 39. Let $A \subseteq \mathbf{G}$ be a set, $\mathrm{E}(A)=|A|^{3} / K$. Suppose that for all $s \neq 0$ the following holds

$$
\left|A_{s}\right| \leqslant \frac{M|A|}{K}
$$

where $M \geqslant 1$ is a real number. Let $K^{4} \leqslant M|A|$. Then there is $s \neq 0$ such that $\left|A_{s}\right| \geqslant$ $|A| / 2 K$ and

$$
\mathrm{E}\left(A_{s}\right) \ll \frac{M^{93 / 79}}{K^{1 / 198}} \cdot\left|A_{s}\right|^{3} .
$$

Proof. Let

$$
P:=\left\{s:\left|A_{s}\right| \geqslant|A| / 2 K\right\} .
$$

Find the number $L$ satisfying $L:=\max _{s \in P}\left|A_{s}\right|^{3} \mathrm{E}^{-1}\left(A_{s}\right)$. In other words for all $s \in P$, one has $\mathrm{E}\left(A_{s}\right) \geqslant\left|A_{s}\right|^{3} / L$. Our task is to find a lower bound for $L$.

Put

$$
\mathcal{C}(x, y)=\mathcal{C}_{3}(A)(x, y):=|A \cap(A-x) \cap(A-y)|
$$

and

$$
\tilde{\mathcal{C}}(x, y)=\mathcal{C}_{4}(A)(x, y, x-y):=|A \cap(A-x) \cap(A-y) \cap(A-x+y)| .
$$

Clearly,

$$
\tilde{\mathcal{C}}(x, y) \leqslant \mathcal{C}(x, y) \leqslant \min \left\{\left|A_{x}\right|,\left|A_{y}\right|\right\}
$$

for any $x, y$. Put also

$$
\mathcal{P}:=\{(x, y): \tilde{\mathcal{C}}(x, y) \geqslant|A| /(4 K L)\}
$$

We will write $\mathcal{P}_{x}:=\mathcal{P} \cap(\{x\} \times \mathbf{G})$, and $\mathcal{P}_{y}:=\mathcal{P} \cap(\mathbf{G} \times\{y\})$. Put also

$$
\mathcal{P}^{\lambda}:=\mathcal{P} \cap\{(x, y): x-y=\lambda\} .
$$

Our first lemma says that the size of $\mathcal{P}$ and some characteristics of the set can be estimated in terms of $L$ and $M$.

Lemma 40. We have

$$
\frac{|A|^{2}}{4 L M^{2}} \leqslant|\mathcal{P}| \leqslant 4 L|A|^{2} .
$$

Further, for any nonzero $y$ and $\lambda$ the following holds

$$
\left|\mathcal{P}_{y}\right|,\left|\mathcal{P}^{\lambda}\right| \leqslant \frac{4 M^{2} L|A|}{K}
$$


Proof. By the Cauchy-Schwarz inequality, we have

$$
\sum_{s \in P}\left|A_{s}\right|^{3} \geqslant 2^{-1} \mathrm{E}_{3}(A) \geqslant 2^{-1}|A|^{4} / K^{2}
$$

Hence

$$
\begin{gathered}
\frac{|A|^{4}}{2 K^{2} L} \leqslant \frac{1}{L} \sum_{s \in P}\left|A_{s}\right|^{3} \leqslant \sum_{s \in P} \mathrm{E}\left(A_{s}\right) \leqslant \sum_{s} \mathrm{E}\left(A_{s}\right)= \\
=\sum_{s} \sum_{z, x, y} A_{s}(z) A_{s}(z+x) A_{s}(z+y) A_{s}(z+x-y)=\sum_{x, y} \tilde{\mathcal{C}}^{2}(x, y) .
\end{gathered}
$$

We can assume that $|A| \geqslant 4 K L^{1 / 2}$ because otherwise the result is trivial in view of the condition $K^{4} \leqslant M|A|$. Since

$$
\sum_{x, y} \tilde{\mathcal{C}}(x, y)=\mathrm{E}(A)=\frac{|A|^{3}}{K}
$$

it follows by (121) and the assumption $|A| \geqslant 4 K L^{1 / 2}$ that

$$
\frac{|A|^{4}}{4 K^{2} L} \leqslant \sum_{0 \neq(x, y) \in \mathcal{P}} \tilde{\mathcal{C}}^{2}(x, y) \leqslant\left(\frac{M|A|}{K}\right)^{2}|\mathcal{P}|
$$

In other words $\frac{|A|^{2}}{4 L M^{2}} \leqslant|\mathcal{P}|$. On the other hand

$$
\frac{|A|}{4 K L}|\mathcal{P}| \leqslant \sum_{(x, y) \in \mathcal{P}} \tilde{\mathcal{C}}(x, y) \leqslant \sum_{x, y} \tilde{\mathcal{C}}(x, y)=\mathrm{E}(A)=\frac{|A|^{3}}{K}
$$

and we obtain the required upper bound for the size of $\mathcal{P}$.

Further, for any fixed $y \neq 0$ the following holds

$$
\left|\mathcal{P}_{y}\right| \leqslant \frac{4 K L}{|A|} \sum_{x} \tilde{\mathcal{C}}(x, y)=\frac{4 K L}{|A|}\left|A_{-y}\right|\left|A_{y}\right| \leqslant \frac{4 M^{2} L|A|}{K} .
$$

Finally,

$$
\left|\mathcal{P}^{\lambda}\right|=\sum_{(x, y): x-y=\lambda} \mathcal{P}(x, y) \leqslant \frac{4 K L}{|A|} \sum_{(x, y): x-y=\lambda} \sum_{x} \tilde{\mathcal{C}}(x, y)=\frac{4 K L}{|A|}\left|A_{\lambda}\right|^{2} \leqslant \frac{4 M^{2} L|A|}{K}
$$

as required.

Now, we show that some norm of $\mathcal{P}$ is huge. Actually, we use the function $\mathcal{C}$ not $\tilde{\mathcal{C}}$ in the proof. 
Lemma 41. One has

$$
\frac{|A|^{5}}{2^{9} K L^{4} M^{6}} \leqslant \sum_{y, x^{\prime}, y^{\prime}}\left|\sum_{x} \mathcal{P}(x, y+x) \mathcal{P}\left(x^{\prime}+x, y^{\prime}+x\right)\right|^{2} .
$$

Proof. As in the proof of Lemma 40, we get

$$
\frac{|A|^{3}}{4 K L M} \leqslant \sum_{(x, y) \in \mathcal{P}} \mathcal{C}(x, y)=\sum_{z} A(z) \sum_{x, y} \mathcal{P}(x, y) A(z+x) A(z+y) .
$$

Using the Cauchy-Schwarz inequality, we obtain

$$
\begin{gathered}
\frac{|A|^{5}}{16 K^{2} L^{2} M^{2}} \leqslant \sum_{z} A(z) \sum_{x, y} \sum_{x^{\prime}, y^{\prime}} \mathcal{P}(x, y) A(z+x) A(z+y) \mathcal{P}\left(x^{\prime}, y^{\prime}\right) A\left(z+x^{\prime}\right) A\left(z+y^{\prime}\right) \leqslant \\
\leqslant \sum_{x, y, x^{\prime}, y^{\prime}} \mathcal{P}(x, y+x) \mathcal{P}\left(x^{\prime}+x, y^{\prime}+x\right) \mathcal{C}_{4}(A)\left(y, x^{\prime}, y^{\prime}\right) .
\end{gathered}
$$

Applying the Cauchy-Schwarz inequality again, we have

$$
\begin{gathered}
\frac{|A|^{10}}{2^{8} K^{4} L^{4} M^{4}} \leqslant \sum_{y, x^{\prime}, y^{\prime}}\left|\sum_{x} \mathcal{P}(x, y+x) \mathcal{P}\left(x^{\prime}+x, y^{\prime}+x\right)\right|^{2} \times \mathrm{E}_{4}(A) \leqslant \\
\leqslant \sum_{y, x^{\prime}, y^{\prime}}\left|\sum_{x} \mathcal{P}(x, y+x) \mathcal{P}\left(x^{\prime}+x, y^{\prime}+x\right)\right|^{2} \times \frac{2 M^{2}|A|^{5}}{K^{3}}
\end{gathered}
$$

because $K^{3} \leqslant K^{4} \leqslant M|A| \leqslant M^{2}|A|$ and hence

$$
\mathrm{E}_{4}(A) \leqslant|A|^{4}+\frac{M^{2}|A|^{2}}{K^{2}} \mathrm{E}(A) \leqslant \frac{2 M^{2}|A|^{5}}{K^{3}} .
$$

This concludes the proof of the lemma.

In terms of the sets $\mathcal{P}^{\lambda}$ we can rewrite expression (124) as

$$
\begin{gathered}
\sum_{y, x^{\prime}, y^{\prime}}\left|\sum_{x} \mathcal{P}(x, y+x) \mathcal{P}\left(x^{\prime}+x, y^{\prime}+x\right)\right|^{2}=\sum_{y, x^{\prime}, y^{\prime}}\left|\sum_{x} \mathcal{P}^{-y}(x) \mathcal{P}^{x^{\prime}-y^{\prime}}\left(x^{\prime}+x\right)\right|^{2}= \\
=\sum_{y, x^{\prime}, y^{\prime}}\left|\sum_{x} \mathcal{P}^{y}(x) \mathcal{P}^{y^{\prime}}\left(x^{\prime}+x\right)\right|^{2}=\sum_{\lambda, \mu} \mathrm{E}\left(\mathcal{P}^{\lambda}, \mathcal{P}^{\mu}\right) .
\end{gathered}
$$

In view of estimate (124), a trivial inequality

$$
\sum_{\lambda, \mu} \mathrm{E}\left(\mathcal{P}^{\lambda}, \mathcal{P}^{\mu}\right) \leqslant \sum_{\lambda, \mu}\left|\mathcal{P}^{\lambda}\right|^{2}\left|\mathcal{P}^{\mu}\right| \ll|\mathcal{P}|^{2} \cdot \max _{\lambda \neq 0}\left|\mathcal{P}^{\lambda}\right|
$$


and bounds for size of $\mathcal{P}^{\lambda}$ of Lemma 40 the sets $\mathcal{P}^{\lambda}$ should look, firstly, like some sets with small doubling (more precisely as sets with large additive energy) and, secondly, the large proportion of such sets must correlate to each other. A model example here is $\mathcal{P}^{\lambda}(x)=Q(x+\alpha(\lambda))$, where $\alpha$ is an arbitrary function and $Q$ is an arithmetic progression of size approximately $\Theta(|A| / K)$. Now we prove that the example is the only case, in some sense.

Put $l=\log (L M)$. Note that $l \leqslant \log (K M)$ because if $L \geqslant K$ then the result is trivial. We can suppose that

$$
2^{17} M^{6} K^{3} L^{7} \leqslant|A|
$$

because otherwise the result follows immediately in view of the condition $M|A| \geqslant K^{4}$. Reducing zero terms, we have

$$
\frac{|A|^{5}}{2^{10} K L^{4} M^{6}} \leqslant \sum_{\lambda, \mu \neq 0} \mathrm{E}\left(\mathcal{P}^{\lambda}, \mathcal{P}^{\mu}\right)
$$

because Lemma 40, estimate (126), a bound

$$
\left|\mathcal{P}^{0}\right| \leqslant 4 K L|A|^{-1} \sum_{x}\left|A_{x}\right|=4 K L|A|
$$

and a calculation

$$
2(4 K L|A|)^{2} 4 L|A|^{2} \leqslant \frac{|A|^{5}}{2^{10} K L^{4} M^{6}} .
$$

Below we will assume that any summation is taken over nonzero indices $\lambda, \mu$. By Lemma 40 and a trivial estimate

$$
\sum_{\lambda}\left|\mathcal{P}^{\lambda}\right|=|\mathcal{P}| \leqslant 4 L|A|^{2}, \quad \mathrm{E}\left(\mathcal{P}^{\lambda}, \mathcal{P}^{\mu}\right) \leqslant\left|\mathcal{P}^{\lambda}\right|\left|\mathcal{P}^{\mu}\right|^{2}
$$

the following holds

$$
\frac{|A|^{5}}{2^{11} K L^{4} M^{6}} \leqslant \sum_{\lambda, \mu:\left|\mathcal{P}^{\lambda}\right|,\left|\mathcal{P}^{\mu}\right| \geqslant \Delta_{*}} \mathrm{E}\left(\mathcal{P}^{\lambda}, \mathcal{P}^{\mu}\right),
$$

where $\Delta_{*}=\frac{|A|}{2^{16} K L^{6} M^{6}}$. Using the pigeonhole principle and Lemma 40, we find a number $\Delta$ such that $\Delta_{*} \leqslant \Delta \leqslant \frac{4 M^{2} L|A|}{K}$ and

$$
\frac{|A|^{5}}{l K L^{4} M^{6}} \ll \sum_{\mu: \Delta<\left|\mathcal{P}^{\mu}\right| \leqslant 2 \Delta} \sum_{\lambda:\left|\mathcal{P}^{\lambda}\right| \geqslant \Delta_{*}} \mathrm{E}\left(\mathcal{P}^{\lambda}, \mathcal{P}^{\mu}\right) .
$$

From (127) and the Cauchy-Schwarz inequality one can see that the summation in the formula is taken over

$$
\mathrm{E}\left(\mathcal{P}^{\mu}\right) \gg \frac{\left|\mathcal{P}^{\mu}\right|^{3}}{l^{2} L^{14} M^{16}}:=\varepsilon\left|\mathcal{P}^{\mu}\right|^{3} .
$$


By inequality (122) of Lemma 40 there is $\mu$ with

$$
\zeta \frac{|A|^{3} \Delta}{K}:=\frac{|A|^{3} \Delta}{l K L^{5} M^{6}} \ll \sum_{\lambda:\left|\mathcal{P}^{\lambda}\right| \geqslant \Delta_{*}} \mathrm{E}\left(\mathcal{P}^{\lambda}, \mathcal{P}^{\mu}\right) .
$$

Put $Q=\mathcal{P}^{\mu}$. We have $\mathrm{E}(Q) \geqslant \varepsilon|Q|^{3}$. Applying a trivial general bound

$$
\mathrm{E}(A, B) \leqslant|A||B| \times \max _{x}|A \cap(B-x)|
$$

we get by Lemma 40

$$
\frac{|A|^{3} \Delta}{l K L^{5} M^{6}} \ll \Delta \frac{4 M^{2} L|A|}{K} \times \sum_{\lambda:\left|\mathcal{P}^{\lambda}\right| \geqslant \Delta_{*}} \max _{x}\left|\mathcal{P}^{\lambda} \cap(Q-x)\right| .
$$

Given an arbitrary $\lambda$ let the maximum in the last formula is attained at point $x:=\alpha(\lambda)$. Thus, we have

$$
\frac{|A|^{2}}{l L^{6} M^{8}} \ll \sum_{\lambda:\left|\mathcal{P}^{\lambda}\right| \geqslant \Delta_{*}}\left|\mathcal{P}^{\lambda} \cap(Q-\alpha(\lambda))\right|=\sum_{\lambda:\left|\mathcal{P}^{\lambda}\right| \geqslant \Delta_{*}} \sum_{x} \mathcal{P}(x, x-\lambda) Q(x+\alpha(\lambda)) .
$$

Hence we find a set $Q$ of the required form, that is having large additive energy and which is correlates with sets $\mathcal{P}^{\lambda}$. Now, we transform the obtained information into some knowledge about the original set $A$.

Using the definition of the set $\mathcal{P}$, we obtain

$$
\begin{gathered}
\frac{|A|^{3}}{l L^{7} M^{8} K} \ll \sum_{x, \lambda} Q(x+\alpha(\lambda)) \sum_{z} A(z) A(z+x) A(z+x-\lambda) A(z+\lambda)= \\
=\sum_{z, \lambda}\left(Q \circ A_{-\lambda}\right)(z-\alpha(\lambda)) A_{\lambda}(z) .
\end{gathered}
$$

We know that $\mathrm{E}(Q) \geqslant \varepsilon|Q|^{3}$. By Balog-Szemerédi-Gowers Theorem 5 we find $Q^{\prime} \subseteq Q$, $\left|Q^{\prime}\right| \gg \varepsilon|Q|$ such that $\left|Q^{\prime}-Q^{\prime}\right| \ll \varepsilon^{-4}\left|Q^{\prime}\right|$. We will prove shortly that the set $Q$ in $(129)$ can be replaced by a set $\tilde{Q}$, namely

$$
c(\varepsilon) \frac{|A|^{3}}{K} \ll \sum_{z, \lambda}\left(\tilde{Q} \circ A_{-\lambda}\right)(z-\alpha(\lambda)) A_{\lambda}(z),
$$

where $c(\varepsilon)>0$ is some constant depends on $L$ and $M$ only and $\tilde{Q}$ has small doubling. Indeed, starting with (128), put $Q_{1}^{\prime}=Q^{\prime}$, and define inductively disjoint sets $Q_{j}^{\prime} \subseteq Q$, $B_{j}=\bigsqcup_{i=1}^{j} Q_{j}^{\prime}, \bar{B}_{j}=Q \backslash B_{j}, j \in[s]$, applying Theorem 5 to $\bar{B}_{j}$. Put also $\mathrm{E}\left(\bar{B}_{j}\right)=\nu_{j}|Q|^{3} \leqslant$ $8 \nu_{j} \Delta^{3}$. If at some stage $j$

$$
\zeta \frac{|A|^{3} \Delta}{2 K}>\sum_{\lambda:\left|\mathcal{P}^{\lambda}\right| \geqslant \Delta_{*}} \mathrm{E}\left(\mathcal{P}^{\lambda}, \bar{B}_{j}\right)
$$


then we stop the algorithm. Let the procedure works exactly $s$ steps and put $B=B_{s}$, $\bar{B}=Q \backslash B$. We claim that $s \ll \varepsilon^{-1}$. To prove this note that if (131) does not hold then

$$
\zeta \frac{|A|^{3} \Delta}{K} \ll \mathrm{E}^{1 / 2}\left(\bar{B}_{j}\right) \sum_{\lambda:\left|\mathcal{P}^{\lambda}\right| \geqslant \Delta_{*}}\left|\mathcal{P}^{\lambda}\right|^{3 / 2} \ll \nu_{j}^{1 / 2} \Delta \frac{M^{2} L|A|}{K} L|A|^{2} .
$$

In other words, $\mathrm{E}\left(\bar{B}_{j}\right) \gg \varepsilon|Q|^{3}$ and, hence, $\left|Q_{j}^{\prime}\right| \gg \varepsilon|Q|,\left|Q_{j}^{\prime}-Q_{j}^{\prime}\right| \ll \varepsilon^{-4}|Q|$. It means, in particular, that after $s \ll \varepsilon^{-1}$ number of steps our algorithm stops indeed. At the last step, we get by the construction that

$$
\begin{gathered}
\zeta \frac{|A|^{3} \Delta}{2 K} \leqslant \sum_{\lambda:\left|\mathcal{P}^{\lambda}\right| \geqslant \Delta_{*}} \mathrm{E}\left(\mathcal{P}^{\lambda}, Q\right)-\sum_{\lambda:\left|\mathcal{P}^{\lambda}\right| \geqslant \Delta_{*}} \mathrm{E}\left(\mathcal{P}^{\lambda}, \bar{B}, \bar{B}\right) \leqslant \\
\left.\leqslant \sum_{\lambda:\left|\mathcal{P}^{\lambda}\right| \geqslant \Delta_{*}} \mathrm{E}\left(\mathcal{P}^{\lambda}, B, B\right)+2 \sum_{\lambda:\left|\mathcal{P}^{\lambda}\right| \geqslant \Delta_{*}} \mathrm{E}\left(\mathcal{P}^{\lambda}, B, \bar{B}\right)\right) .
\end{gathered}
$$

Let us prove the following estimate

$$
\zeta \frac{|A|^{3} \Delta}{K} \ll \sum_{\lambda:\left|\mathcal{P}^{\lambda}\right| \geqslant \Delta_{*}} \mathrm{E}\left(\mathcal{P}^{\lambda}, B\right) .
$$

If not then by the Cauchy-Schwarz inequality and the choice of $\bar{B}$, we obtain

$$
\left(\zeta \frac{|A|^{3} \Delta}{K}\right)^{2} \ll\left(\sum_{\lambda:\left|\mathcal{P}^{\lambda}\right| \geqslant \Delta_{*}} \mathrm{E}\left(\mathcal{P}^{\lambda}, B, \bar{B}\right)\right)^{2} \leqslant \sum_{\lambda:\left|\mathcal{P}^{\lambda}\right| \geqslant \Delta_{*}} \mathrm{E}\left(\mathcal{P}^{\lambda}, B\right) \cdot \zeta \frac{|A|^{3} \Delta}{2 K}
$$

and we get a contradiction. Hence the following holds

$$
\zeta \frac{|A|^{3} \Delta}{K} \ll \sum_{\lambda:\left|\mathcal{P}^{\lambda}\right| \geqslant \Delta_{*}} \mathrm{E}\left(\mathcal{P}^{\lambda}, B\right) .
$$

Applying the Hölder inequality, we find a set $Q_{j}^{\prime}$ such that

$$
\zeta \frac{|A|^{3} \Delta}{s^{2} K} \ll \sum_{\lambda:\left|\mathcal{P}^{\lambda}\right| \geqslant \Delta_{*}} \mathrm{E}\left(\mathcal{P}^{\lambda}, Q_{j}^{\prime}\right) .
$$

So, putting $Q^{\prime}:=Q_{j}^{\prime}$ we get $(130)$ with $c(\varepsilon) \gg \frac{\zeta \varepsilon^{2}}{l L^{2} M^{2}}$. Of course, the summation in the obtained formula can be taken just over $\lambda$ with $\left|A_{\lambda}\right| \gg c(\varepsilon) \frac{|A|}{K}$ and we will assume this.

Denote by $\Omega$ the set

$$
\Omega:=\left\{(z, \lambda): A_{\lambda}(z)=1, \text { and }\left(\tilde{Q} \circ A_{-\lambda}\right)(z-\alpha(\lambda)) \geqslant \frac{c(\varepsilon)|A|}{2 K}\right\} .
$$


From (130) and our assumption (119) we have $|\Omega| \gg c(\varepsilon) M^{-1}|A|^{2}$. On the other hand, considering $\Omega_{\lambda}:=\{z:(z, \lambda) \in \Omega\}$ for any fixed $\lambda$, one has

$$
\left|\Omega_{\lambda}\right| \frac{|A|}{K} c(\varepsilon) \ll \sum_{z} A_{\lambda}(z)\left(\tilde{Q} \circ A_{-\lambda}\right)(z-\alpha(\lambda)) \leqslant\left|A_{\lambda}\right||Q| \ll\left|A_{\lambda}\right| \Delta \ll \frac{M^{3} L|A|^{2}}{K^{2}} .
$$

Hence there are at least $\gg c^{2}(\varepsilon) K M^{-4} L^{-1}|A|$ sets $A_{\lambda}$ such that there exists some $z=z(\lambda)$ with $\left(\tilde{Q} \circ A_{\lambda}\right)(z-\alpha(\lambda)) \gg c(\varepsilon)|A| / 2 K$. Denote the set of these $\lambda$ by $T$. For any such $A_{\lambda}$ there exists a shift of the set $\tilde{Q}$ such that $\left|A_{\lambda} \cap(\tilde{Q}+w(\lambda))\right| \gg c(\varepsilon)|A| / 2 K \gg_{\varepsilon, M}\left|A_{\lambda}\right|$. Put $A_{\lambda}^{\prime}:=A_{\lambda} \cap(\tilde{Q}+w(\lambda))$. We have by Lemma 4 that for any $\lambda_{1}, \lambda_{2} \in T$ the following holds

$$
\left|A_{\lambda_{1}}^{\prime}+A_{\lambda_{2}}^{\prime}\right| \leqslant|\tilde{Q}+\tilde{Q}| \ll \varepsilon^{-8} \frac{M^{2} L|A|}{K} .
$$

In particular,

$$
\mathrm{E}\left(A_{\lambda_{1}}^{\prime}, A_{\lambda_{2}}^{\prime}\right) \gg \frac{c^{4}(\varepsilon) \varepsilon^{8}|A|^{3}}{M^{2} L K^{3}} .
$$

Finally, using (125) as well as Lemma 7 with $k=l=2$, we obtain

$$
\begin{gathered}
\frac{|A|^{5}}{l^{64} M^{458} L^{395} K} \ll \frac{\zeta^{8} \varepsilon^{24}|A|^{5}}{l^{8} M^{26} L^{19} K} \ll \frac{c^{8}(\varepsilon) \varepsilon^{8}|A|^{5}}{M^{10} L^{3} K} \ll|T|^{2} \cdot \frac{c^{4}(\varepsilon) \varepsilon^{8}|A|^{3}}{M^{2} L K^{3}} \ll \sum_{\lambda_{1}, \lambda_{2} \in T} \mathrm{E}\left(A_{\lambda_{1}}^{\prime}, A_{\lambda_{2}}^{\prime}\right) \leqslant \\
\leqslant \sum_{\lambda_{1}, \lambda_{2} \in T} \mathrm{E}\left(A_{\lambda_{1}}, A_{\lambda_{2}}\right) \leqslant \mathrm{E}_{4}(A) \leqslant \frac{2 M^{2}|A|^{5}}{K^{3}}
\end{gathered}
$$

with the required lower bound for $L$. This completes the proof of Theorem 39 .

We finish the section by analog of Definition 11, which we will use in the the last part of the paper.

Definition 42. For $\beta, \gamma \in[0,1]$ a set $A$ is called $U^{k}(\beta, \gamma)$-connected if for any $B \subseteq A$, $|B| \geqslant \beta|A|$ the following holds

$$
\|B\|_{\mathcal{U}^{k}} \geqslant \gamma\left(\frac{|B|}{|A|}\right)^{2^{k}}\|A\|_{\mathcal{U}^{k}}
$$

Again, if, say, $\gamma^{-1}|A|^{8} /|A \pm A|^{4} \geqslant\|A\|_{\mathcal{U}^{3}}$ then by inequality (115) one can see that $A$ is $U^{3}(\beta, \gamma)$-connected for any $\beta$. The existence of $U^{k}(\beta, \gamma)$-connected subsets in an arbitrary set is discussed in the Appendix. 


\section{Self-dual sets}

Inequality (115) gives us a relation between $\|A\|_{\mathcal{U}^{3}}$ and $\mathrm{E}(A)$. It attaints at a random subset $A$ of $\mathbf{G}$, where by randomness we mean that each element of $A$ belongs to the set with probability $\mathrm{E}(A) /|A|^{3}$. On the other hand, it is easy to see that an upper bound takes place

$$
\|A\|_{\mathcal{U}^{3}}=\sum_{s} \mathrm{E}\left(A_{s}\right) \leqslant \sum_{s}\left|A_{s}\right|^{3}=\mathrm{E}_{3}(A) .
$$

A weaker estimate follows from (132) combining with the Cauchy-Schwarz inequality

$$
\|A\|_{\mathcal{U}^{3}}^{2} \leqslant \mathrm{E}_{4}(A) \mathrm{E}(A) .
$$

In the section we consider sets having critical relations between $\|A\|_{\mathcal{U}^{3}}$ and $\mathrm{E}_{4}(A), \mathrm{E}(A)$ that is the sets satisfying the reverse inequality to (133) (actually, we use just a slightly stronger estimate then reverse to (132)). It turns out that they are exactly which we called self-dual sets.

Let us recall a result on large deviations. The following variant can be found in [7].

Lemma 43. Let $X_{1}, \ldots, X_{n}$ be independent random variables with $\mathbb{E} X_{j}=0$ and $\mathbb{E}\left|X_{j}\right|^{2}=$ $\sigma_{j}^{2}$. Let $\sigma^{2}=\sigma_{1}^{2}+\cdots+\sigma_{n}^{2}$. Suppose that for all $j \in[n]$, we have $\left|X_{j}\right| \leqslant 1$. Let also a be a real number such that $\sigma^{2} \geqslant 6$ na. Then

$$
\mathbb{P}\left(\left|\frac{X_{1}+\cdots+X_{n}}{n}\right| \geqslant a\right) \leqslant 4 e^{-n^{2} a^{2} / 8 \sigma^{2}} .
$$

We need in a combinatorial lemma.

Lemma 44. Let $\Delta, \sigma, C>1$ are positive numbers, $t$ be a positive integer, and $M_{1}, \ldots, M_{t}$ be sets, $\Delta \leqslant\left|M_{j}\right| \leqslant C \Delta, j \in[t], \sigma \leqslant 10^{-4} t^{2} \Delta$, where

$$
\sigma:=\sum_{i, j=1}^{t}\left|M_{i} \bigcap M_{j}\right| .
$$

Then there are at least $\frac{t^{2} \Delta}{16(2 C+1) \sigma}$ disjoint sets $\tilde{M}_{l} \subseteq M_{i_{l}}$ such that $\left|\tilde{M}_{l}\right| \geqslant \frac{\Delta}{4(2 C+1)}$.

Proof. We will choose our sets $\tilde{M}_{i}$ deterministically from a randomly chosen family. The family will be taken with probability at least $1 / 4$.

First of all, we note that

$$
10^{-4} t^{2} \Delta \geqslant \sigma \geqslant \sum_{i=1}^{t}\left|M_{i}\right| \geqslant t \Delta .
$$

Put $p=t \Delta 2^{-1} \sigma^{-1}$. In view of (134), we get $p \in(0,1 / 2]$. Let us form a new family of sets taking a set $M_{i}$ from $M_{1}, \ldots, M_{t}$ uniformly and independently with probability $p$. Denote 
the obtained family as $M_{1}^{\prime}, \ldots, M_{s}^{\prime}$. By Lemma 43 and bound (134), we have after some calculations that

$$
2^{-1} p t \leqslant s \leqslant 2 p t
$$

with probability at least $3 / 4$. Further the expectation of $\sigma$ equals

$$
\begin{aligned}
& \mathbb{E} \sum_{i, j=1}^{t}\left|M_{i} \bigcap M_{j}\right|=\sum_{x} \sum_{i=1}^{t} \mathbb{E} M_{i}(x)+\sum_{x} \sum_{i, j=1, i \neq j}^{t} \mathbb{E} M_{i}(x) M_{j}(x)= \\
& =p \sum_{i=1}^{t}\left|M_{i}\right|+p^{2} \sum_{i, j=1, i \neq j}^{t}\left|M_{i} \bigcap M_{j}\right| \leqslant C p t \Delta+p^{2} \sigma \leqslant(2 C+1) p^{2} \sigma
\end{aligned}
$$

by our choice of $p$. Hence, by Markov inequality, with probability at least $1 / 2$ one has

$$
\sum_{i, j=1}^{s}\left|M_{i}^{\prime} \bigcap M_{j}^{\prime}\right| \leqslant(4 C+2) p^{2} \sigma
$$

and by the Cauchy-Schwarz inequality, we get

$$
\left|\bigcup_{i=1}^{s} M_{i}^{\prime}\right| \geqslant \frac{\left(\sum_{i=1}^{s}\left|M_{i}^{\prime}\right|\right)^{2}}{\sum_{i, j=1}^{s}\left|M_{i}^{\prime} \cap M_{j}^{\prime}\right|} \geqslant \frac{s^{2} \Delta^{2}}{(4 C+2) p^{2} \sigma} \geqslant \frac{2 s^{2} \sigma}{(2 C+1) t^{2}}:=q .
$$

Now find disjoint subsets $\tilde{M}_{i} \subseteq M_{i}^{\prime}, i \in[s]$, taking it consequently and using greedy choice. Thus, we have at most $s$ nonempty sets $\tilde{M}_{i}$ such that $\bigsqcup_{i} \tilde{M}_{i}=\bigcup_{i=1}^{s} M_{i}^{\prime}$. Hence

$$
2 \sum_{i:\left|\tilde{M}_{i}\right| \geqslant q(2 s)^{-1}}\left|\tilde{M}_{i}\right| \geqslant \sum_{i}\left|\tilde{M}_{i}\right|=\left|\bigcup_{i=1}^{s} M_{i}^{\prime}\right| \geqslant q .
$$

By our choice of parameters and estimates (135) the following holds

$$
\left|\tilde{M}_{i}\right| \geqslant \frac{q}{2 s}=\frac{s \sigma}{(2 C+1) t^{2}} \geqslant \frac{p \sigma}{(4 C+2) t}=\frac{\Delta}{(8 C+4)} .
$$

Similarly, the number $n$ of the sets $\tilde{M}_{i}$ can be estimated from (136)

$$
n \geqslant \frac{q}{2 \Delta}=\frac{s^{2} \sigma}{(2 C+1) t^{2} \Delta} \geqslant \frac{p^{2} \sigma}{(8 C+4) \Delta}=\frac{t^{2} \Delta}{(32 C+16) \sigma} .
$$

This completes the proof.

Let us remark an interesting consequence of Lemma 44 .

Corollary 45. Let $A \subseteq \mathbf{G}$ be a $(2, \beta, \gamma)$-connected set, $\beta \leqslant 0.5$ be a constant. Then there is a set $P \subseteq\{x:(A \circ A)(x) \geqslant \Delta\}$ satisfies $\mathrm{E}^{P}(A) \gg \mathrm{E}(A) \log ^{-1}|A|$, and there are $k \gg \gamma|A| \Delta^{-1} \log ^{-1}|A|$ disjoint sets $\tilde{A}_{j} \subseteq A_{s_{j}}$ with $\left|\tilde{A}_{j}\right| \gg \Delta$. 
Proof. Using Lemma 13, we find $A^{\prime},\left|A^{\prime}\right| \geqslant|A| / 2$ such that estimate (33) takes place. We want to apply Lemma 44 to the sets $A_{s}^{\prime} \subseteq A_{s}, s \in P^{\prime}$, where $P^{\prime}=\left\{x:\left(A^{\prime} \circ A^{\prime}\right)(x) \sim \Delta\right\}$, $\mathrm{E}^{P^{\prime}}\left(A^{\prime}\right) \gg \mathrm{E}\left(A^{\prime}\right) \log ^{-1}|A|$. Of course, such set $P^{\prime}$ exists by the pigeonhole principle. To apply Lemma 44, we need to calculate the quantity $\sigma$

$$
\sigma:=\sum_{s, t \in P^{\prime}}\left|A_{s}^{\prime} \bigcap A_{t}^{\prime}\right|=\sum_{x, y} \mathcal{C}_{3}\left(A^{\prime}\right)(x, y) P^{\prime}(x) P^{\prime}(y)
$$

By the last identity and estimate (33) (for details, see [33]), we get

$$
\sigma \ll \frac{\mathrm{E}(A)}{|A|} \cdot\left|P^{\prime}\right| .
$$

Applying Lemma 44, we find disjoint sets $\tilde{A}_{j} \subseteq A_{s_{j}}^{\prime} \subseteq A_{s_{j}}, s_{j} \in P^{\prime}, j \in[k]$ such that

$$
k \gg \frac{\left|P^{\prime}\right|^{2} \Delta|A|}{\mathrm{E}(A)\left|P^{\prime}\right|} \gg \gamma \frac{|A|}{\Delta \log |A|} .
$$

In the last inequality we have used $(2, \beta, \gamma)$-connectedness of $A$. To complete the proof note that $P^{\prime} \subseteq\{x:(A \circ A)(x) \geqslant \Delta\}$.

Clearly, the bound on $k$ in Corollary 45 is the best possible up to logarithms. Calculating $\mathrm{E}\left(A, A_{j}\right) /\left|A_{j}\right|$ and comparing its with $\mathrm{E}_{3}$ (see [33]) one can obtain an alternative proof of lower bounds for $\left|A \pm A_{s}\right|$ as of section 5. Another result on a family of disjoint $A_{s}$ is proved in Proposition 49 below.

Now we are able to obtain the main result of the section.

Theorem 46. Let $A \subseteq \mathbf{G}$ be a set, and $M \geqslant 1$ be a real number. Put $l=\log |A|$. Suppose that $A$ is $U^{3}(\beta, \gamma)$ and $(2, \beta, \gamma)$-connected with $\beta \leqslant 0.5$. Then inequality

$$
\|A\|_{\mathcal{U}^{3}}^{2} \gg_{M} \mathrm{E}_{4}(A) \mathrm{E}(A)
$$

takes place iff there is a positive real $\Delta \sim_{M, l} \mathrm{E}_{3}(A) \mathrm{E}(A)^{-1}$ and a set

$$
P \subseteq\left\{s \in A-A: \Delta<\left|A_{s}\right|\right\}
$$

such that $|P| \gg_{M, l}|A|, P=-P$, further,

$$
\mathrm{E}^{P}(A) \gg_{M, l} \mathrm{E}(A), \quad \mathrm{E}_{3}^{P}(A) \gg_{M, l} \mathrm{E}_{3}(A), \quad \mathrm{E}_{4}^{P}(A) \gg_{M, l} \mathrm{E}_{4}(A) .
$$

and such that for any $s \in P$ there is $H^{s} \subseteq A_{s},\left|H^{s}\right| \gg_{M, l} \Delta$, with

$$
\left|H^{s}-H^{s}\right| \ll_{M, l}\left|H^{s}\right|,
$$

and $\mathrm{E}\left(A, H^{s}\right) \ll_{M, l}\left|H^{s}\right|^{3}$.

Moreover there are disjoint sets $H_{j} \subseteq A_{s_{j}},\left|H_{j}\right| \gg_{M, l} \Delta, s_{j} \in P, j \in[k]$ such that all $H_{j}$ have small doubling property (139), $\mathrm{E}\left(A, H_{j}\right) \ll_{M, l}\left|H_{j}\right|^{3}$ and $k \gg_{M, l}|A| \Delta^{-1}$. 
Proof. Put $a=|A|, \mathrm{E}=\mathrm{E}(A), \mathrm{E}_{3}=\mathrm{E}_{3}(A), \mathrm{E}_{4}=\mathrm{E}_{4}(A)$. Let us begin with the necessary condition. Using Lemma 13 , we find $A^{\prime},\left|A^{\prime}\right| \geqslant|A| / 2$ such that estimate (33) takes place. Because of $A$ is $U^{3}(\beta, \gamma)$ and $(2, \beta, \gamma)$-connected with $\beta \leqslant 0.5$, we have $\left\|A^{\prime}\right\|_{\mathcal{U}^{3}} \sim\|A\|_{\mathcal{U}^{3}}$ and $\mathrm{E}\left(A^{\prime}\right) \sim \mathrm{E}(A)$. Combining assumption (137) with the Cauchy-Schwarz inequality, we get

$$
\|A\|_{\mathcal{U}^{3}}^{2} \gg_{M} \mathrm{E}_{4}(A) \mathrm{E}(A) \geqslant \mathrm{E}_{3}^{2}(A)
$$

In particular, by the last inequality and (132), (133), we obtain

$$
\begin{gathered}
\mathrm{E}_{3}^{2}\left(A^{\prime}\right) \geqslant\left\|A^{\prime}\right\|_{\mathcal{U}^{3}}^{2} \gg\|A\|_{\mathcal{U}^{3}}^{2} \geqslant_{M} \mathrm{E}_{3}^{2}(A), \\
\mathrm{E}_{4}\left(A^{\prime}\right) \mathrm{E}\left(A^{\prime}\right) \geqslant\left\|A^{\prime}\right\|_{\mathcal{U}^{3}}^{2} \gg\|A\|_{\mathcal{U}^{3}}^{2} \geqslant_{M} \mathrm{E}_{4}(A) \mathrm{E}(A) \geqslant \mathrm{E}_{4}(A) \mathrm{E}\left(A^{\prime}\right)
\end{gathered}
$$

and, hence, $\mathrm{E}_{3}\left(A^{\prime}\right) \sim_{M} \mathrm{E}_{3}(A), \mathrm{E}_{4}\left(A^{\prime}\right) \sim_{M} \mathrm{E}_{4}(A)$. With some abuse of the notation we will use the same letter $A$ for $A^{\prime}$ below. By Lemma 7, we have

$$
\|A\|_{\mathcal{U}^{3}}=\sum_{s} \mathrm{E}\left(A_{s}\right)=\sum_{s} \sum_{t}\left(A_{s} \circ A_{s}\right)^{2}(t) \gg_{M} \sum_{s}\left|A_{s}\right|^{3}=\sum_{s} \mathrm{E}\left(A, A_{s}\right)=\mathrm{E}_{3} .
$$

One can assume that the summation in the last formula is taken over $s$ such that $\left|A_{s}\right| \gg_{M}$ $\mathrm{E}_{3} \mathrm{E}^{-1}$ and $\mathrm{E}\left(A_{s}\right) \gg_{M}\left|A_{s}\right|^{3},\left|A_{s}\right|^{3} \gg_{M} \mathrm{E}\left(A, A_{s}\right)$. Let us consider the condition $\mathrm{E}\left(A_{s}\right) \gg_{M}$ $\left|A_{s}\right|^{3}$. By Balog-Szemerédi-Gowers Theorem we can find $H^{s} \subseteq A_{s}$ with $\left|H^{s}\right| \gg_{M}\left|A_{s}\right|$, and $\left|H^{s}-H^{s}\right| \ll_{M}\left|H^{s}\right|$. Loosing a logarithm $l=\log a$ we can assume that the summation in (141) is taken over $\left|A_{s}\right|, \Delta<\left|A_{s}\right| \leqslant 2 \Delta, \Delta \gg_{M, l} \mathrm{E}_{3} \mathrm{E}^{-1}$ and $\mathrm{E}\left(A_{s}\right) \gg_{M, l}\left|A_{s}\right|^{3}$, $\left|A_{s}\right|^{3} \gg_{M, l} \mathrm{E}\left(A, A_{s}\right)$. By $P$ denote the set of such $s$. Thus, $|P| \Delta^{3} \gg_{M, l} \mathrm{E}_{3}$ and it is easy to check that $P=-P$. Note also that $\mathrm{E}\left(A, H^{s}\right) \ll_{M, l}\left|H^{s}\right|^{3}$ for any $s \in P$. We have

$$
\sum_{s, t \in P}\left(A_{s} \circ A_{s}\right)^{2}(t) \gg_{M} \mathrm{E}_{3}
$$

Returning to (140), we obtain

$$
\left(|P| \Delta^{3}\right)^{2} \gg_{M, l} \max \left\{|P| \Delta^{4} \mathrm{E}, \mathrm{E}_{4}|P| \Delta^{2}\right\}
$$

and hence $|P| \Delta^{2} \gg_{M, l} \mathrm{E},|P| \Delta^{4} \gg_{M, l} \mathrm{E}_{4}$. Thus, $\Delta \sim_{M, l} \mathrm{E}_{3} \mathrm{E}_{2}^{-1}$ and

$$
\mathrm{E}_{4} \sim_{M, l} \Delta \mathrm{E}_{3} \sim_{M, l} \Delta^{2} \mathrm{E} \sim_{M, l} \Delta^{4}|P|
$$

So, we know all energies $\mathrm{E}, \mathrm{E}_{3}, \mathrm{E}_{4}$ if we know $|P|$ and $\Delta$. Let us estimate the size of the $P$. Taking any $s \in P$, we get by Lemma 13 that

$$
\Delta^{3} \ll_{M, l} \mathrm{E}\left(A_{s}\right) \leqslant \mathrm{E}\left(A, A_{s}\right) \ll \mathrm{E} a^{-1} \Delta \ll_{M, l}|P| \Delta^{3} a^{-1}
$$

or $|P| \gg_{M} a$. So, we have proved (138)—(139).

Further

$$
\sum_{s, t \in P}\left|H^{s} \cap H^{t}\right| \leqslant \sum_{s, t \in P}\left|A_{s} \cap A_{t}\right|:=\sigma .
$$


Applying Lemma 44, we find disjoint sets $H_{j} \subseteq A_{s_{j}},\left|H_{j}\right| \gg_{M}\left|A_{s_{j}}\right|,\left|H_{j}-H_{j}\right| \leqslant \mid H_{j}-$ $H_{j}\left|\ll_{M, l}\right| H_{j}|,| H_{j} \mid \gg \Delta \sim_{M, l} \mathrm{E}_{3}(A) \mathrm{E}(A)^{-1}, j \in[k]$ and $k \gg|P|^{2} \Delta \sigma^{-1}$. Arguing as in Corollary 45, we get $\sigma \ll \mathrm{E}|P||A|^{-1}$ and hence $k \gg|P| \Delta|A| \mathrm{E}^{-1} \gg_{M, l}|A| \Delta^{-1}$. Of course the last bound on $k$ is the best possible up to constants depending on $M, l$. We have obtained the necessary condition.

Let us prove the sufficient condition. Using the Cauchy-Schwarz inequality and formulas (138)-(139), we have

$$
\begin{gathered}
\|A\|_{\mathcal{U}^{3}}^{2} \geqslant\left(\sum_{s \in P} \mathrm{E}\left(A_{s}\right)\right)^{2} \geqslant\left(\sum_{s \in P} \mathrm{E}\left(H_{s}\right)\right)^{2} \gg_{M, l}\left(\sum_{s \in P}\left|H_{s}\right|^{3}\right)^{2} \gg_{M, l} \\
\gg_{M, l}|P|^{2} \Delta^{6} \gg_{M, l} \mathrm{E}(A) \mathrm{E}_{4}(A)
\end{gathered}
$$

as required. This completes the proof.

Remark 47. In the statement of Theorem 46 there is the set of popular differences $P$ and the structure of $A$ is described in terms of the set $P$. Although, we have obtained a criterium it can be named as a weak structural result. Perhaps, a stronger version avoiding using of the set $P$ takes place. Namely, under the hypothesis of Theorem 46 there are disjoint sets $H_{j} \subseteq A_{s_{j}},\left|H_{j}\right| \gg_{M, l} \Delta, \Delta \sim_{M, l} \mathrm{E}_{3}(A) \mathrm{E}(A)^{-1}, j \in[k]$ such that (139) holds and

$$
\sum_{j=1}^{k}\left|H_{j}\right|^{4} \gg_{M, l} \mathrm{E}_{3}(A), \quad \sum_{j=1}^{k}\left|H_{j}\right|^{3} \gg_{M, l} \mathrm{E}(A), \quad \sum_{j=1}^{k}\left|H_{j}\right|^{5} \gg_{M, l} \mathrm{E}_{4}(A) .
$$

It is easy to see that it is a sufficient condition. Indeed, because the sets $H_{j} \subseteq A$ are disjoint, we have

$$
\|A\|_{\mathcal{U}^{3}} \geqslant \sum_{j=1}^{k}\left\|H_{j}\right\|_{\mathcal{U}^{3}}
$$

Using the assumption $\left|H_{j}-H_{j}\right| \ll_{M, l}\left|H_{j}\right|$, the first bound from (143), as well as Corollary 38 , we obtain

$$
\|A\|_{\mathcal{U}^{3}} \gg_{M, l} \sum_{j=1}^{k}\left|H_{j}\right|^{4} \gg_{M, l} \mathrm{E}_{3}(A)
$$

and, similarly, by the second and the third inequality of (143), we get

$$
\|A\|_{\mathcal{U}^{3}}^{2} \gg_{M, l} \mathrm{E}(A) \mathrm{E}_{4}(A)
$$

as required. 
Example 48. Let $A \subseteq \mathbf{G}$ be a set having small Wiener norm, that is the following quantity $\|A\|_{W}:=N^{-1} \sum_{\xi}|\widehat{A}(\xi)|:=M$ is small. Then for any $B \subseteq A$, applying the Parseval identity, one has

$$
|B|=\sum_{x} B(x) A(x)=N^{-1} \sum_{\xi} \widehat{B}(\xi) \overline{\widehat{A}(\xi)} .
$$

Using the Hölder inequality twice (see also [19]), we get

$$
|B|^{4} \leqslant\left(N^{-1} M \sum_{\xi}|\widehat{B}(\xi)|^{2}|\widehat{A}(\xi)|\right)^{2} \leqslant M^{2}|B| \mathrm{E}(A, B)
$$

or, in other words,

$$
\mathrm{E}(A, B) \geqslant \frac{|B|^{3}}{M^{2}} .
$$

By the multiplicative property of Wiener norm, we have $\left\|A_{s}\right\|_{W} \leqslant M^{2}$. In particular, $\mathrm{E}\left(A_{s}\right) \geqslant \frac{\left|A_{s}\right|^{3}}{M^{4}}$. Hence $\|A\|_{\mathcal{U}^{3}} \geqslant M^{-4} \mathrm{E}_{3}(A)$. Further, $\mathrm{E}(A) \geqslant M^{-2}|A|^{3}, \mathrm{E}_{3}(A) \geqslant M^{-4}|A|^{4}$ and hence

$$
\|A\|_{\mathcal{U}^{3}}^{2} \geqslant M^{-8} \mathrm{E}_{3}^{2}(A) \geqslant M^{-16} \mathrm{E}(A) \mathrm{E}_{4}(A) .
$$

Thus, an application of Theorem 46 gives us that $A$ has very explicit structure $(2-$ connectedness follows from (144) and $U^{3}$-connectedness can be obtained via formula (118) in a similar way). Another structural result on sets from $\mathbb{F}_{p}$ with small Wiener norm was given in [19].

If Theorem 39 does not hold that is $\mathrm{E}\left(A_{s}\right) \gg\left|A_{s}\right|^{3}$ for all $s$ then, clearly, $\|A\|_{\mathcal{U}^{3}} \gg$ $\mathrm{E}_{3}(A)$ and we can try to apply our structural Theorem 46 . On the other hand, if $A$ is a self-dual set, that is a disjoint union of sets with small doubling then for any $s \neq 0$ one has exactly $\mathrm{E}\left(A_{s}\right) \gg\left|A_{s}\right|^{3}$. It does not contradict to Theorem 39 because condition (119).

Roughly speaking, in the proof of Theorem 46 we found disjoint subsets of $A_{s}$, containing huge amount of the energy (see also Corollary 45). One can ask about the possibility to find some number of disjoint $A_{s}$ (and not its subsets) in general situation. Our next statement answer the question affirmatively.

Proposition 49. Let $A \subseteq \mathbf{G}$ be a set, $D \subseteq A-A$. Put

$$
\sigma:=\sum_{s \in D}\left|A-A_{s}\right|
$$

Then there are at least $l \geqslant|D|^{2} /(4 \sigma)$ disjoint sets $A_{s_{1}}, \ldots, A_{s_{l}}$. In particular, if

$$
\left|A^{2}-\Delta(A)\right| \leqslant \frac{|A-A|^{2}}{M}
$$

then there are at least $l \geqslant M / 4$ disjoint sets $A_{s_{1}}, \ldots, A_{s_{l}}$. 
Proof. Our arguments is a sort of an algorithm. By (145) there is $s_{1} \in D$ such that $\left|A-A_{s_{1}}\right| \leqslant \sigma /|D|$. Put $D_{1}=D \backslash\left(A-A_{s_{1}}\right)$. If $\left|D_{1}\right|<|D| / 2$ then terminate the algorithm. If not then by an obvious estimate

$$
\sum_{s \in D_{1}}\left|A-A_{s}\right| \leqslant \sigma
$$

we find $s_{2} \in D_{1}$ such that

$$
\left|A-A_{s_{2}}\right| \leqslant \frac{\sigma}{\left|D_{1}\right|} \leqslant \frac{2 \sigma}{|D|} .
$$

Put $D_{2}=D_{1} \backslash\left(A-A_{s_{2}}\right)$. If $\left|D_{1}\right|<|D| / 2$ then terminate the algorithm. And so on. At the last step, we obtain the set $D_{l}=D \backslash \bigcup_{j=1}^{l}\left(A-A_{s_{j}}\right),\left|D_{l}\right|<|D| / 2$. It follows that

$$
\frac{|D|}{2} \leqslant\left|\bigcup_{j=1}^{l}\left(A-A_{s_{j}}\right)\right| \leqslant \sum_{j=1}^{l}\left|A-A_{s_{j}}\right| \leqslant l \frac{2 \sigma}{|D|} .
$$

Thus $l \geqslant|D|^{2} /(4 \sigma)$. Finally, recall that

$$
t \in A-A_{s} \quad \text { iff } \quad A_{t} \cap A_{s} \neq \emptyset .
$$

Thus all constructed sets $A_{s_{1}}, \ldots, A_{s_{l}}$ are disjoint.

To get (146) put $D=A-A$ and recall that by Lemma 10 the following holds $\mid A^{2}-$ $\Delta(A)\left|=\sum_{s \in A-A}\right| A-A_{s} \mid$. This completes the proof.

One can ask is it true that not only $\mathrm{E}_{3}$ energy but $U^{3}$-norm of sumsets or difference sets is large? It is easy to see that the answer is no, because of our basic example $A=H \dot{+} \Lambda$, $|\Lambda|=K$. In the case $\mathrm{E}(A) \sim|A|^{3} / K, \mathrm{E}_{3}(A) \sim|A|^{4} / K$ but $\|A\|_{\mathcal{U}^{3}} \sim|A|^{4} / K^{2}$ and similar for $A \pm A$.

\section{Appendix}

In the section we prove that any set contains a relatively large connected subset. The case $k=2$ of Proposition 55 below was proved in [31] (with slightly worse constants) and we begin with a wide generalization.

Definition 50. Let $X, Y$ be two nonempty sets, $|X|=|Y|$. A nonnegative symmetric function $q(x, y), x \in X, y \in Y$ is called weight if the correspondent matrix $q(x, y)$ is nonnegatively defined.

Having two sets $A$ and $B$ put $\mathrm{E}^{q}(A, B):=\sum_{x, y} q(x, y) A(x) B(y), \mathrm{E}^{q}(A):=\mathrm{E}^{q}(A, A)$. Clearly, $\mathrm{E}^{q}(A, B) \leqslant|A||B|\|q\|_{\infty}$. The main property of any weight is the following.

Lemma 51. Let $q$ be a weight. Then for any sets $A, B$, one has

$$
\left(\mathrm{E}^{q}(A, B)\right)^{2} \leqslant \mathrm{E}^{q}(A) \mathrm{E}^{q}(B) .
$$


Example 52. Clearly, the function $q(x, y)=(B \circ B)^{k}(x-y)$ for any set $B$ and an arbitrary positive integer $k$ is a weight. Further, by the construction of Gowers $U^{d}$-norms it follows that

$$
q_{d}\left(x_{1}, x_{1}^{\prime}\right)=\sum_{x_{2}, \ldots, x_{d} \in \mathbf{G}} \sum_{x_{2}^{\prime}, \ldots, x_{d}^{\prime} \in \mathbf{G}} \prod_{\omega \in\{0,1\}^{d}} f\left(\operatorname{pr}\left(\vec{x}^{\omega}\right)\right)
$$

is also a weight for any nonnegative function $f$. In formula (148), we have $\vec{x}=\left(x_{1}, \ldots, x_{d}\right)$, $\vec{x}^{\prime}=\left(x_{1}^{\prime}, \ldots, x_{d}^{\prime}\right)$, and $\operatorname{pr}\left(y_{1}, \ldots, y_{d}\right):=y_{1}+\cdots+y_{d}$. Another example of a weight is

$$
q_{d}^{*}(x, y)=\sum_{h_{1}, \ldots, h_{d-1}} \prod_{\omega \in\{0,1\}^{d-1}, \omega \neq 0} f(x+\omega \cdot \vec{h}) f(y+\omega \cdot \vec{h}),
$$

where $f$ is an arbitrary nonnegative function again and $\vec{h}=\left(h_{1}, \ldots, h_{d-1}\right)$.

For two sets $S, T \subseteq \mathbf{G}, S \neq \emptyset, T \subseteq S$ put $\mu_{S}(T)=|T| /|S|$. Now we prove a general lemma on connected sets and quantities $\mathrm{E}^{q}$, where $q$ is a weight.

Lemma 53. Let $A, B \subseteq \mathbf{G}$ be two sets, $\beta_{1}, \beta_{2}, \rho \in(0,1]$ be real numbers, $\beta_{1} \leqslant \beta_{2}$, $\rho<\beta_{1} / \beta_{2}$. Let $q$ be a weight. Suppose that $\mathrm{E}^{q}(A) \geqslant c|A|^{2}\|q\|_{\infty}, c \in(0,1]$. Then there is $A^{\prime} \subseteq A$ such that for any subset $\tilde{A} \subseteq A^{\prime}, \beta_{1}\left|A^{\prime}\right| \leqslant|\tilde{A}| \leqslant \beta_{2}\left|A^{\prime}\right|$ one has

$$
\mathrm{E}^{q}(\tilde{A}) \geqslant \rho^{2} \mu_{A^{\prime}}^{2}(\tilde{A}) \cdot \mathrm{E}^{q}\left(A^{\prime}\right),
$$

and besides

$$
\mathrm{E}^{q}\left(A^{\prime}\right)>\left(1-\beta_{2} \rho\right)^{2 s} \mathrm{E}^{q}(A),
$$

where $s \leqslant \log (1 / c)\left(2 \log \left(\frac{1-\beta_{2} \rho}{1-\beta_{1}}\right)\right)^{-1}$.

Proof. Put $b=\|q\|_{\infty}$. We use an inductive procedure in the proof. Let us describe the first step of our algorithm. Suppose that (150) does not hold for some set $C \subseteq A$, $\beta_{1}|A| \leqslant|C| \leqslant \beta_{2}|A|$. Put $A^{1}=A \backslash C$. Then $\left|A^{1}\right| \leqslant\left(1-\beta_{1}\right)|A|$. Using Lemma 51, we get

$$
\mathrm{E}^{q}(A)=\mathrm{E}^{q}(C, A)+\mathrm{E}^{q}\left(A^{1}, A\right)<\rho \mu_{A}(C) \mathrm{E}^{q}(A)+\mathrm{E}_{q}^{1 / 2}\left(A^{1}\right) \mathrm{E}_{q}^{1 / 2}(A) .
$$

Hence

$$
\mathrm{E}^{q}\left(A^{1}\right)>\mathrm{E}^{q}(A)\left(1-\mu_{A}(C) \rho\right)^{2} \geqslant \mathrm{E}^{q}(A)\left(1-\beta_{2} \rho\right)^{2} .
$$

After that applying the same arguments to the set $A^{1}$, find a subset $C \subseteq A^{1}$ such that (150) does not hold (if it exists) and so on. We obtain a sequence of sets $A \supseteq A^{1} \supseteq \cdots \supseteq A^{s}$, and $\left|A^{s}\right| \leqslant\left(1-\beta_{1}\right)^{s}|A|$. So, at the step $s$, we have

$$
c|A|^{2} b\left(1-\beta_{2} \rho\right)^{2 s} \leqslant \mathrm{E}^{q}(A)\left(1-\beta_{2} \rho\right)^{2 s}<\mathrm{E}^{q}\left(A^{s}\right) \leqslant\left|A^{s}\right|^{2} b \leqslant\left(1-\beta_{1}\right)^{2 s}|A|^{2} b .
$$

Thus, our algorithm must stop after at most $s \leqslant \log (1 / c)\left(2 \log \left(\frac{1-\beta_{2} \rho}{1-\beta_{1}}\right)\right)^{-1}$ number of steps. Putting $A^{\prime}=A^{s}$, we see that inequality (150) takes place for any $\tilde{A} \subseteq A^{\prime}$ with $\beta_{1}\left|A^{\prime}\right| \leqslant|\tilde{A}| \leqslant \beta_{2}\left|A^{\prime}\right|$. Finally, by the second estimate in (152), we obtain (151). This concludes the proof. 
Let us formulate a useful particular case of Lemma 53.

Lemma 54. Let $A, B \subseteq \mathbf{G}$ be two sets, $\beta_{1}, \beta_{2}, \rho \in(0,1]$ be real numbers, $\beta_{1} \leqslant \beta_{2}$, $\rho<\beta_{1} / \beta_{2}$. Suppose that $\mathrm{E}(A, B) \geqslant c|A|^{2}|B|, c \in(0,1]$. Then there is $A^{\prime} \subseteq A$ such that for any subset $\tilde{A} \subseteq A^{\prime}, \beta_{1}\left|A^{\prime}\right| \leqslant|\tilde{A}| \leqslant \beta_{2}\left|A^{\prime}\right|$ one has

$$
\mathrm{E}(\tilde{A}, B) \geqslant \rho^{2} \mu_{A^{\prime}}^{2}(\tilde{A}) \cdot \mathrm{E}\left(A^{\prime}, B\right)
$$

and besides

where $s \leqslant \log (1 / c)\left(2 \log \left(\frac{1-\beta_{2} \rho}{1-\beta_{1}}\right)\right)^{-1}$.

$$
\mathrm{E}\left(A^{\prime}, B\right)>\left(1-\beta_{2} \rho\right)^{2 s} \mathrm{E}(A, B)
$$

Lemma 54 implies the required statement, generalizing the result from [31].

Proposition 55. Let $A \subseteq \mathbf{G}$ be a set, $\beta \in(0,1)$ be real numbers, and $k \geqslant 2$ be an integer. Put $c=\mathrm{E}_{k}(A)|A|^{-(k+1)}$. Then there is $A^{\prime} \subseteq A$ such that

$$
\mathrm{E}_{k}\left(A^{\prime}, A\right)>\left(1-2^{-1} \beta\right)^{2 s} \mathrm{E}_{k}(A),
$$

where $s \leqslant \log (1 / c)\left(2 \log \left(\frac{2-\beta}{2-2 \beta}\right)\right)^{-1}$, and $A^{\prime}$ is $(k, \beta, \gamma)$-connected with

$$
\gamma \geqslant 2^{-(2 s k+2 k-2 s)} \beta^{2 k}(2-\beta)^{2 s(k-1)} .
$$

In particular,

$$
\left|A^{\prime}\right| \geqslant\left(1-2^{-1} \beta\right)^{s} c^{1 / 2}|A|
$$

Proof. Note that $T \subseteq S$ iff $\Delta(T) \subseteq \Delta(S)$. Applying Lemma 54 with $A=\Delta(A), B=$ $A^{k-1}, \beta_{1}=\beta, \beta_{2}=1, \rho=\beta_{1} /\left(2 \beta_{2}\right)=\beta / 2$, and using formula (17), we find a set $A^{\prime} \subseteq A$ such that for any subset $\tilde{A} \subseteq A^{\prime}, \beta\left|A^{\prime}\right| \leqslant|\tilde{A}|$ one has

$$
\mathrm{E}_{k}(\tilde{A}, A) \geqslant \rho^{2} \mu_{A^{\prime}}^{2}(\tilde{A}) \cdot \mathrm{E}_{k}\left(A^{\prime}, A\right),
$$

and

$$
\mathrm{E}_{k}\left(A^{\prime}, A\right)>\left(1-2^{-1} \beta\right)^{2 s} \mathrm{E}_{k}(A),
$$

where $s \leqslant \log (1 / c)\left(2 \log \left(\frac{1-\rho}{1-\beta}\right)\right)^{-1}$. We have obtained inequality (155). From (158), (159) and the Hölder inequality, we get

$$
\begin{gathered}
\mathrm{E}_{k}(\tilde{A}) \geqslant \rho^{2 k} \mu_{A^{\prime}}^{2 k}(\tilde{A}) \mathrm{E}_{k}^{k}\left(A^{\prime}, A\right) \mathrm{E}_{k}^{-(k-1)}(A) \geqslant\left(2^{-1} \beta\right)^{2 k}\left(1-2^{-1} \beta\right)^{2 s(k-1)} \mu_{A^{\prime}}^{2 k}(\tilde{A}) \mathrm{E}_{k}\left(A^{\prime}, A\right) \geqslant \\
\geqslant\left(2^{-1} \beta\right)^{2 k}\left(1-2^{-1} \beta\right)^{2 s(k-1)} \mu_{A^{\prime}}^{2 k}(\tilde{A}) \mathrm{E}_{k}\left(A^{\prime}\right) .
\end{gathered}
$$

Thus, the set $A^{\prime}$ is $(k, \beta, \gamma)$-connected with $\gamma$ satisfying (156). To obtain (157) just apply (159) and a trivial upper bound for $\mathrm{E}_{k}\left(A^{\prime}, A\right)$

$$
\left|A^{\prime}\right|^{2}|A|^{k-1} \geqslant \mathrm{E}_{k}\left(A^{\prime}, A\right)>\left(1-2^{-1} \beta\right)^{2 s} \mathrm{E}_{k}(A)=\left(1-2^{-1} \beta\right)^{2 s} c|A|^{k+1}
$$

as required. This completes the proof.

In view of Lemma 53 and Example 52 one can obtain an analog of Proposition 55 for $U^{k}(\beta, \gamma)$-connected sets, see Definition 42 . We leave the details to an interested reader. 


\section{Acknowledgements}

The author is grateful to Vsevolod F. Lev and Tomasz Schoen for useful discussions.

\section{References}

[1] N. Alon, V. Rödl. Sharp bounds for some multicolor Ramsey numbers. Combinatorica, 25:125-141, 2005.

[2] T. Austin. On the Norm Convergence of Nonconventional Ergodic Averages. Ergodic Theory and Dynamical Systems, 30:321-338, 2010.

[3] M. Bateman, N. Katz. New bounds on cap sets. J. Amer. Math. Soc., 25(2):585-613, 2012.

[4] M. Bateman, N. Katz. Structure in additively nonsmoothing sets. arXiv:1104.2862v1.

[5] B. Bollobás, A. Thomason. Projections of bodies and hereditary properties of hypergraphs. Bull. London Math. Soc., 27:417-424, 1995.

[6] H. Furstenberg. Recurrence in ergodic theory and combinatorial number theory, Princeton N.J., 1981.

[7] B. Green. Arithmetic Progressions in Sumsets. Geom. Funct. Anal., 12(3):584-597, 2002.

[8] B. Green, T. Tao. The primes contain arbitrarily long arithmetic progressions. Annals of Math. 167(2):481-547, 2008.

[9] B. Green, T. Tao. An inverse theorem for the Gowers $U^{3}(G)$ norm. Proc. Edinb. Math. Soc. (2), 51(1):73-153, 2008.

[10] B. Green, T. Tao. The quantitative behaviour of polynomial orbits on nilmanifolds. Ann. of Math. (2), 175(2):465-540, 2012.

[11] B. Green, T. Tao. The equivalence between inverse sumset theorems and inverse conjectures for the $U^{3}$-norm. Math. Proc. Cambridge Philos. Soc., 149(1):1-19, 2010.

[12] B. Green, T. Tao. Quadratic uniformity for the Möbius function. Ann. Inst. Fourier (Grenoble), 58(6):1863-1935, 2008.

[13] B. Green, T. Tao. The quantitative behaviour of polynomial orbits on nilmanifolds. Ann. of Math.(2), 175(2):465-540, 2012.

[14] W.T. Gowers. A new proof of Szemerédi's theorem for arithmetic progressions of length four. Geom. Func. Anal., 8:529-551, 1998.

[15] W.T. Gowers. A new proof of Szemerédi's theorem. Geom. Funct. Anal., 11:465-588, 2001.

[16] B. Host, B. Kra. Nonconventional ergodic averages and nilmanifolds. Ann. of Math. (2), 161(1):397-488, 2005.

[17] B. Host, B. Kra. Convergence of polynomial ergodic averages. Israel J. Math., 149:1-19, 2005. 
[18] N. H. Katz and P. Koester. On additive doubling and energy. SIAM J. Discrete Math., 24:1684-1693, 2010.

[19] S.V. Konyagin, I.D. Shkredov. On Wiener norm of subsets of $\mathbb{Z}_{p}$ of medium size. arXiv:1403.8129v1.

[20] S. Lovett, R. Meshulam, A. Samorodnitsky. Inverse Conjecture for the Gowers norm is false. Theory Comput., 7:131-145, 2011.

[21] B. Murphy, O. Roche-Newton and I.D. Shkredov. Variations on the sum-product problem. arXiv:1312.6438v2.

[22] G. Petridis. New proofs of Plünnecke-type estimates for product sets in groups. Combinatorica, 1-14, 2012.

[23] W. Rudin. Fourier analysis on groups. Wiley 1990 (reprint of the 1962 original).

[24] A. Samorodnitsky, L. Trevisan. Gowers uniformity, influence of variables, and PCPs. SIAM J. Comput., 39(1):323-360, 2009.

[25] T. Sanders. On the Bogolyubov-Ruzsa lemma. Anal. PDE, 5(3):627-655, 2012.

[26] T. Sanders. Approximate (abelian) groups. arXiv:1212.0456 [math.CA].

[27] T. Schoen. New bounds in Balog-Szemerédi-Gowers theorem. preprint.

[28] T. Schoen and I. Shkredov. Higher moments of convolutions. J. Number Theory, 133(5):1693-1737, 2013.

[29] T. Schoen and I. Shkredov. Additive properties of multiplicative subgroups of $\mathbb{F}_{p}$. $Q$. J. Math., 63(3):713-722, 2012.

[30] T. Schoen and I. Shkredov. On sumsets of convex sets. Combin. Probab. Comput., 20(5):793-798, 2011.

[31] I. Shkredov. On Sets with Small Doubling. Mat. Zametki, 84(6):927-947, 2008.

[32] I. Shkredov. Some new inequalities in additive combinatorics. MJCNT, 3(2):237-288, 2013.

[33] I. Shkredov. Some new results on higher energies. Transactions of $M M S, 74(1): 35-73$, 2013.

[34] I. Shkredov and I. Vyugin. On additive shifts of multiplicative subgroups. Sb. Math., 203(5-6):844-863, 2012.

[35] T. Tao. A quantitative ergodic theory proof of Szemerédi's theorem. Electron. J. Combin., 13(1), Research Paper 99, 2006.

[36] T. Tao. Norm convergence of multiple ergodic averages for commuting transformations. Ergodic Theory Dynam. Systems, 28(2):657-688, 2008.

[37] T. Tao and V. Vu. Additive Combinatorics. Cambridge University Press, 2006.

[38] T. Tao, T. Ziegler. The inverse conjecture for the Gowers norm over finite fields via the correspondence principle. Anal. PDE, 3(1):1-20, 2010.

[39] T. Ziegler. A non-conventional ergodic theorem for a nilsystem. Ergodic Theory Dynam. Systems, 25(4):1357-1370, 2005.

[40] T. Ziegler. Universal characteristic factors and Furstenberg averages. J. Amer. Math. Soc., 20(1):53-97, 2007. 